\title{
Mutually exclusive signaling signatures define the hepatic and pancreatic progenitor cell lineage divergence
}

\author{
Elisa Rodríguez-Seguel, ${ }^{1}$ Nancy Mah, ${ }^{2}$ Heike Naumann, ${ }^{1}$ Igor M. Pongrac, ${ }^{1}$ Nuria Cerdá-Esteban, ${ }^{1}$ \\ Jean-Fred Fontaine, ${ }^{2}$ Yongbo Wang, ${ }^{3}$ Wei Chen, ${ }^{3}$ Miguel A. Andrade-Navarro, ${ }^{2}$ \\ and Francesca $M$. Spagnoli ${ }^{1,4}$ \\ ${ }^{1}$ Laboratory of Molecular and Cellular Basis of Embryonic Development, ${ }^{2}$ Computational Biology and Data Mining, ${ }^{3}$ Laboratory \\ for Novel Sequencing Technology, Functional and Medical Genomics, Max Delbrück Center for Molecular Medicine, D-13092 \\ Berlin, Germany
}

Understanding how distinct cell types arise from multipotent progenitor cells is a major quest in stem cell biology. The liver and pancreas share many aspects of their early development and possibly originate from a common progenitor. However, how liver and pancreas cells diverge from a common endoderm progenitor population and adopt specific fates remains elusive. Using RNA sequencing (RNA-seq), we defined the molecular identity of liver and pancreas progenitors that were isolated from the mouse embryo at two time points, spanning the period when the lineage decision is made. The integration of temporal and spatial gene expression profiles unveiled mutually exclusive signaling signatures in hepatic and pancreatic progenitors. Importantly, we identified the noncanonical Wnt pathway as a potential developmental regulator of this fate decision and capable of inducing the pancreas program in endoderm and liver cells. Our study offers an unprecedented view of gene expression programs in liver and pancreas progenitors and forms the basis for formulating lineage-reprogramming strategies to convert adult hepatic cells into pancreatic cells.

[Keywords: liver; pancreas; mouse; RNA-seq; Wnt signaling]

Supplemental material is available for this article.

Received April 18, 2013; revised version accepted July 29, 2013.

The liver and pancreas share many common aspects of their early embryonic development as well as some adult functional properties and both are endowed with essential metabolic functions (Slack 2007; Zaret 2008). This close relationship makes the liver a very attractive tissue source for generating new pancreatic $\beta$ cells by lineage reprogramming, which might be used in the context of cell-based therapy of diabetes. However, how liver and pancreas cells diverge from a common endoderm progenitor population and adopt specific fates remains elusive.

During embryonic development, the pancreas originates from distinct outgrowths of the dorsal and ventral regions of the foregut endoderm (Spagnoli 2007; Puri and Hebrok 2010). Subsequently, the two buds fuse to form a single organ containing both pancreatic exocrine and endocrine cells (Spagnoli 2007; Puri and Hebrok 2010).

${ }^{4}$ Corresponding author

E-mail francesca.spagnoli@mdc-berlin.de

Article is online at http://www.genesdev.org/cgi/doi/10.1101/gad.220244.113.
The liver originates solely from the ventral foregut endoderm, adjacent to where the ventral pancreas (vpa) emerges, and the two cell fates are specified concomitantly by mouse embryonic day 8.5 (E8.5) (Deutsch et al. 2001). Previous studies showed that the ventral foregut endoderm is multipotent for the hepatic and pancreatic programs (Deutsch et al. 2001; Tremblay and Zaret 2005; Miki et al. 2012). Hepatic and pancreatic endoderm express a common set of transcription factors, such as the FoxA and GATA factors, and are exposed to the same extrinsic signals: fibroblast growth factor (FGF) and BMP (Deutsch et al. 2001; Chung et al. 2008; Spagnoli and Brivanlou 2008; Zaret 2008). However, the developmental regulators of the fate choice between liver and pancreas are poorly understood. It is also unknown how the pancreatic program is stably repressed in nascent hepatic progenitors and how the hepatic program is repressed in pancreatic progenitors. This knowledge will aid in not only the programming of stem cells to pancreatic and hepatic lineages, but also the discovery of the 
mechanisms underlying cellular plasticity between liver and pancreas.

Studies conducted on various stem cell types or whole organisms (Tumbar et al. 2004; Wang et al. 2004; Dequéant et al. 2006; Mitiku and Baker 2007) have clearly demonstrated that significant insights into the regulation of the cell fate decision are obtained through global analysis of gene expression events. Thus, to search for developmental regulators that are involved in the control of the pancreas and liver cell fate decision, it is fundamental to have a global picture of the regulation of gene expression in the mammalian hepatic-pancreatic endoderm lineage. To this aim, we performed sequencing-based expression profiling (RNA sequencing [RNA-seq]) on hepatic and pancreatic progenitors in mice at distinct developmental stages. We devised a strategy to purify liver and pancreas progenitor populations directly from the endoderm of $\mathrm{Tg}$ (Prox1-EGFP [enhanced green fluorescent protein]) reporter mouse embryos before and after the onset of organogenesis. By integrating the temporal and spatial gene expression profiles, we found mutually exclusive signaling signatures in hepatic and pancreatic progenitors. Importantly, we identified the noncanonical Wnt pathway as a potential developmental regulator of the pancreas versus liver fate decision, since it is expressed in the foregut endoderm before the cell fate choice is made and then is maintained in pancreas progenitors but is absent in hepatic progenitors. Moreover, when assayed in Xenopus embryos and mammalian cells, activation of the noncanonical Wnt pathway is able to promote pancreatic fate, suggesting an ancient mechanism for controlling the pancreas versus liver fate choice. Overall, our study offers an unprecedented view of gene expression programs in liver and pancreas progenitors during the defined period of their lineage divergence and provides novel insights into key mechanisms that may underpin cellular plasticity and reprogramming between the two cell types.

\section{Results \\ RNA-seq on FACS-purified hepatic and pancreatic progenitors}

To explore the transcription program associated with liver and pancreas progenitors in vivo at the time of their fate divergence, we performed RNA-seq on distinct mouse endoderm progenitor populations isolated at two developmental stages. For in vivo monitoring of hepatic and pancreatic progenitor cells, we used the transgenic mouse line Tg(Prox1-EGFP) $)^{\text {Gsat/Mmcd }}$ that carries the reporter gene EGFP into the Prox 1 homeobox gene locus (Gong et al. 2003). Prox1 is the earliest specific marker in common between hepatic and pancreatic endoderm from gastrulation onward (Burke and Oliver 2002; Wandzioch and Zaret 2009) and therefore is ideally suited for isolating both hepatic and pancreatic progenitors (Fig. 1). We showed that Proxl-EGFP transgenic mouse embryos reproduced the endogenous pattern of Proxl expression in the endoderm from E7.5 onward (Fig. 1A,B; Burke and Oliver 2002; data not shown). In both ventral and dorsal foregut endoderm of $\mathrm{Tg}$ (Prox1-EGFP) embryos, the localization of EGFP perfectly mirrored endogenous Prox1 expression and overlapped with other endodermal genes, such as Foxa2, at E8.5 and with tissue-specific genes, such as Pdx1 in the pancreas or Liv2 in the liver, from E9.5 onward (Fig. 1A,B; Watanabe et al. 2002; Lee et al. 2005; Wandzioch and Zaret 2009; Puri and Hebrok 2010). Thus, the $\mathrm{Tg}$ (Prox1-EGFP) in vivo model enabled us to visualize hepatic and pancreatic progenitors under fluorescence microscopy before organogenesis had started. Distinct regions of the prospective hepatic and pancreatic endoderm were manually microdissected, and Prox1-EGFP ${ }^{+}$ cells were FACS-purified and subjected to RNA-seq analysis to define their transcriptomes (Fig. 1C-E). Previous fate mapping studies in mouse and chick embryos suggested differential locations of hepatic and pancreatic progenitors in the ventral foregut at the early somite stage (Tremblay and Zaret 2005; Miki et al. 2012). To determine whether regional identity within the foregut is associated with differential gene expression, we collected Prox1$\mathrm{EGFP}^{+}$cells of the whole ventral foregut (referred to as fg) and exclusively of the medial ventral foregut (referred to as $\mathrm{mfg}$ ) from E8.5 embryos at the same somite stage (seven to nine somites) (Fig. 1C, top). At E10.5, budding sites of the liver and pancreas, including both the dorsal pancreas (dpa) and vpa, were readily distinguishable, and the three distinct progenitor populations were isolated and processed for the analysis (Fig. 1C, bottom).

Given the small number of progenitor cells, we applied a submicrogram RNA-seq method and used paired-end sequencing technology (Adamidi et al. 2011). A comparable number of high-quality raw reads was obtained from each sample and used to estimate the relative abundance of transcripts (Table 1; Trapnell et al. 2010). Principal component analysis (PCA) on the nonredundant gene expression data showed that individual samples perfectly clustered according to their embryonic stage and/or region of origin (Fig. 2A). Known hepatic and pancreatic tissue-specific transcripts were found in the E10.5 liver and pancreas RNA-seq profiles, respectively, reflecting the high reliability and accuracy of the data obtained (Fig. 1E; Supplemental Fig. 1).

\section{Integrating temporal and spatial transcription profiles of the progenitor populations}

To identify developmental regulators of pancreatic and/or hepatic fate, we integrated the temporal and spatial analyses. Both hepatic and pancreatic progenitors arise from foregut endoderm cells (Spagnoli 2007; Zaret 2008). Therefore, the set of genes expressed in the foregut (fg or $\mathrm{mfg}$ ) is expected to include regulators of both early pancreatic and hepatic fate specification as well as the choice between the two fates (liver and pancreas). We compared those genes with the genes expressed later in the E10.5 liver, vpa, and dpa and found a number of genes specific to either liver or pancreas that were already expressed at the earlier time point in the E8.5 foregut (89 and 517 genes, respectively) (Fig. 2B, left). Of interest, these numbers suggest a higher divergence between the 
A
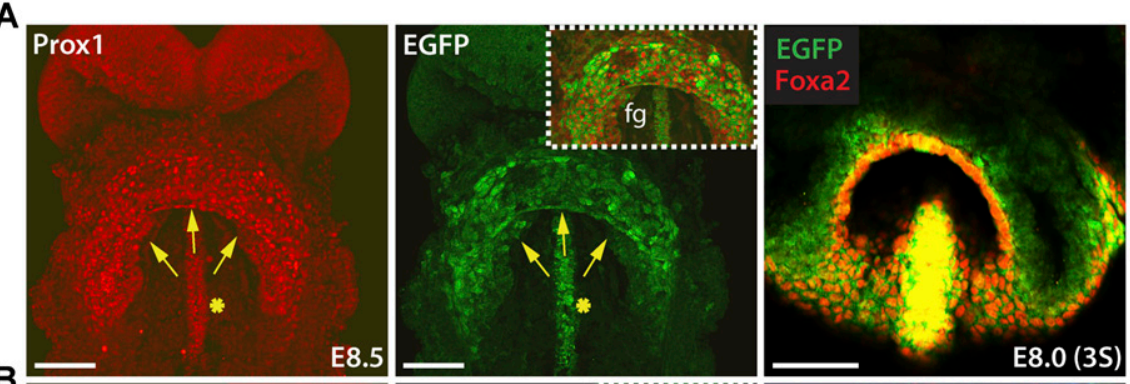

Prox1
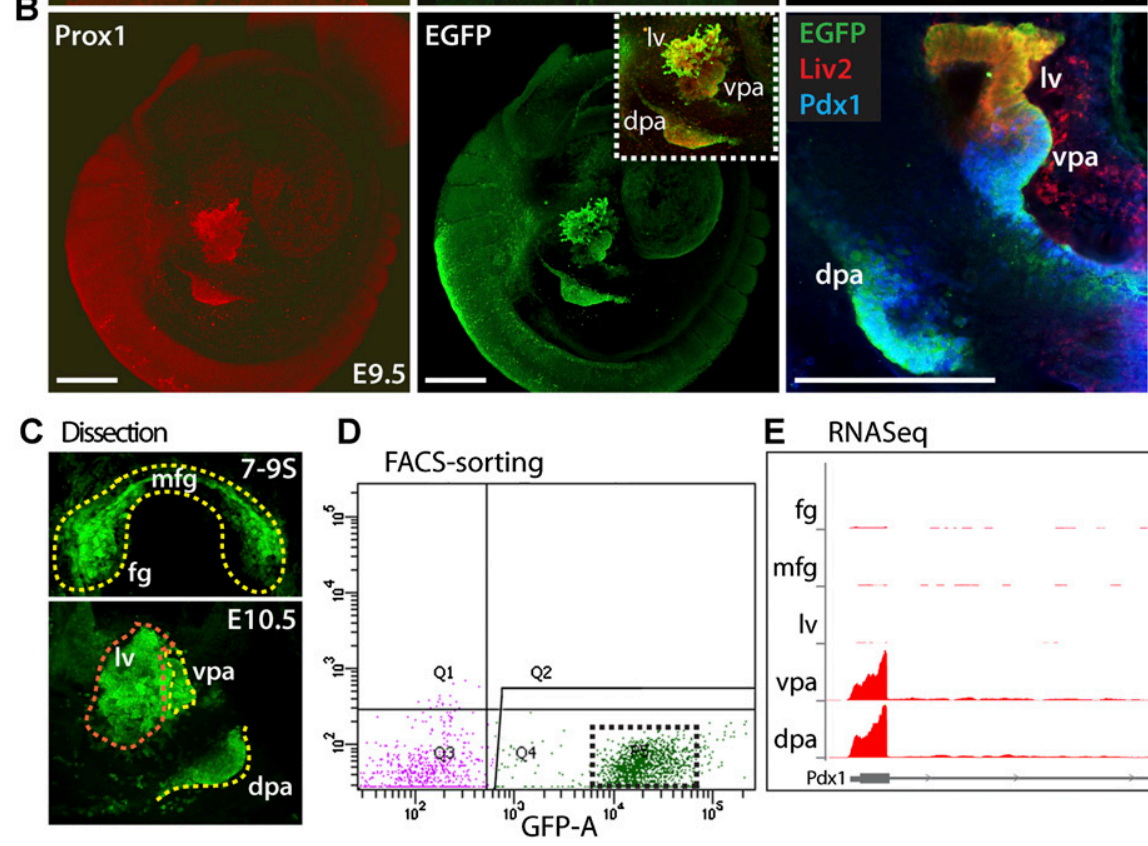

E RNASeq

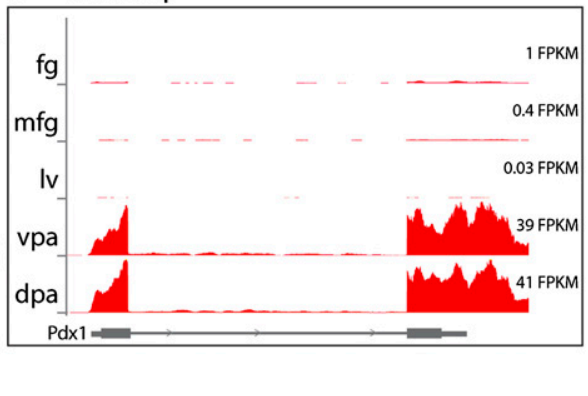

Figure 1. In vivo isolation and RNA-seq profiling of endoderm progenitor cells from $\operatorname{Tg}(\operatorname{Prox} 1$-EGFP) mouse embryos. $(A)$ Representative maximum confocal Z-projections of immunofluorescence analysis in Tg(Prox1-EGFP) E8.5 mouse embryos shows EGFP reporter expression in both ventral (arrows) and dorsal (asterisk) foregut cells, mirroring endogenous Proxl expression (see the inset for overlay) and overlapping with Foxa2 expression domains at the three-somite (3S) stage. Embryos are presented in ventral view. (B) In Tg(Prox1-EGFP) E9.5 mouse embryos, EGFP expression was detected in the hepatic and both dorsal and ventral pancreatic buds, mirroring the endogenous Prox1 (see the inset for overlay). EGFP colocalized with Pdx1 in both pancreatic buds and with Liv2 solely in the liver bud. Embryos are presented in lateral view. $(C-E)$ Schematic representation of cell sampling and the RNA-seq procedure. $(C) \mathrm{EGFP}^{+}$fg and $\mathrm{mfg}$ endoderm at the seven- to nine-somite (7-9S) stage/E8.5 and liver (lv), ventral (vpa), and dorsal (dpa) pancreas at E10.5 were microdissected from $\mathrm{Tg}$ (Proxl-EGFP) embryos. Cells were dissociated and subjected to FACS. $(D)$ Representative diagram of the $\mathrm{EGFP}^{+}$cell fraction isolated by FACS. The dashed box indicates EGFP ${ }^{+}$-gated cells, and cells negative for EGFP are in purple. Transcript expression was profiled by RNA-seq. (E) Example illustrating the RNA-seq read coverage profile of the pancreatic-specific gene $P d \times 1$. The $Y$-axis indicates the number of read counts in each cell population ( $Y$-axis scale is 01000 counts). FPKM (fragments per kilobase of exon per million fragments mapped) values for $P d x 1$ in each data set are included on the right. Exons are depicted as gray boxes at the bottom on the $X$-axis. As expected, a large number of reads was found in pancreatic progenitors. Bars: $A, 100 \mu \mathrm{m} ; B, 50 \mu \mathrm{m}$.

foregut and liver when compared with the number of genes whose expression is instead maintained in the pancreas (vpa and dpa) (Fig. 2B, left; Supplemental Fig. 2). This is in line with the hypothesis of the pancreas being the default fate of the ventral foregut endoderm (Deutsch et al. 2001). In contrast, genes initially showing low expression in the foregut but high expression later in E10.5 hepatic or pancreatic progenitors represent cell type-specific markers of differentiation and possibly factors involved in late aspects of organogenesis (Fig. 2B, right; Supplemental Fig. 3). At this later time point, the number of genes that are unique to either the dpa or vpa
(211 vs. 257) (Fig. 2B, right) indicates a large divergence between the two buds, reflecting the dual embryological origin of the pancreas.

\section{Different Wnt signaling signatures correlate with pancreatic or hepatic progenitor states}

To study the mechanisms underlying the pancreas versus liver fate decision, we focused on the transcripts whose expression was detected in the E8.5 foregut and maintained exclusively in the pancreatic rudiments and therefore possibly was endowed with a role in this cell fate 
Table 1. Summary of sequencing data and annotation information

\begin{tabular}{llccc}
\hline & & \multicolumn{2}{c}{ Number of transcripts $^{\mathbf{a}}$} \\
\cline { 4 - 5 } Sample & Raw reads & Mapped reads & Annotated & Unannotated \\
\hline fg & $121,497,737$ & $115,767,182$ & 38,159 & 19,348 \\
mfg & $114,786,211$ & $108,295,227$ & 38,254 & 19,347 \\
Liver & $114,436,606$ & $109,182,827$ & 38,260 & 19,363 \\
vpa & $109,534,481$ & $102,391,351$ & 38,171 & 19,371 \\
dpa & $104,526,851$ & $99,464,506$ & 38,168 & 19,356 \\
\hline
\end{tabular}

The transcript abundance was calculated by estimating the fragments per kilobase of exon per million mapped fragments (FPKM). An average of 38,000 annotated distinct transcripts per sample was detected.

${ }^{\text {a}}$ This includes all Cufflinks genes found in each sample before filtering. Annotated transcripts were considered to be all Cufflinks genes that were associated to a gene symbol.

restriction. We hereafter refer to this cluster as FP (foregut pancreas) (Fig. 2B, left). A gene ontology (GO) term analysis of our results revealed significant enrichment for Wntrelated "Molecular Function" categories in the group FP, such as Wnt protein-binding (GO:0017147; seven genes; $P$-value $<0.00001 .9)$ and Wnt-activated receptor (GO: 0042813; five genes; $P$-value $<0.00035)$ activities. Among the Wnt-associated factors were genes such as Frizzled 2 (Fzd2), Fzd4, Ror2, Sfrp5, Sfrp2, and Wls (Supplemental Table 1). Also, Wnt-related "Biological Process" categories were primarily enriched in the group FP, such as establishment of epithelial cell polarity (GO: 0090162), Wnt receptor signaling pathway (GO: 0007223), and establishment of planar polarity (GO: 0001736) (Supplemental Table 2). Of interest, no other regions of the Venn diagram displayed such a significant enrichment for Wnt-related categories as the group FP, suggesting a unique signaling signature of the pancreas versus liver fate in the mammalian endoderm.

We decided to further characterize the expression dynamics of Wnt signal transducers. Interestingly, in our data set, we found that intracellular transducers of the canonical Wnt/ $\beta$-catenin signaling (Grigoryan et al. 2008; Clevers and Nusse 2012)-such as $\beta$-catenin (Ctnnb1), $A P C, A x i n$, and TCFs - as well as the common canonical and noncanonical component Dishevelled 1 (Dvl1) (Angers and Moon 2009; Kikuchi et al. 2009/ were expressed at stable levels in the different progenitor populations (Fig. 2C), whereas transcripts encoding ligands, receptors, and coreceptors exhibited lineage-specific induction or repression (Fig. 2D). In particular, only noncanonical Wnt ligands (Wnt5a, Wnt5b, and Wnt7b) (Kikuchi et al. 2009; Grumolato et al. 2010) were found in the foregut and pancreas and all of them were strongly down-regulated in liver progenitors (Supplemental Fig. 4). Additional determinants of noncanonical/planar cell polarity (PCP) Wnt specificity displayed similar expression profiles, including the receptors Fzd2 and Fzd7 and the PCP core membrane proteins Stbm/Vangl2, Celsr2, and Fat1 (Fig. 2D). Finally, the coreceptors Lrp5 and Ror2 showed divergent expressions-one strongly induced in the liver, and the other one induced exclusively in pancreas progenitors
(Fig. 2D). These results suggest a cell type-dependent noncanonical Wnt activation in the foregut and pancreatic progenitors that is ensured by not only distinct classes of ligands, but also recruitment of unrelated coreceptors, as previously described in other contexts /Grumolato et al. 2010). For instance, the differential expression of Ror2 coreceptor might sustain noncanonical Wnt activation in foregut and pancreas progenitors but not in liver progenitors.

\section{Noncanonical Wnt signaling signature underlies the pancreas versus liver fate decision}

If multiple components of a pathway are expressed and the expression is developmentally regulated, it is very likely that the pathway is active. Therefore, we undertook several approaches to study the possible role of the Wnt noncanonical pathway in the process of pancreas versus liver cell fate specification. For further analysis, we prioritized FP group transcripts that showed significant differential regulation between the pancreatic and hepatic endoderm (150 transcripts out of 517; Cufflinks, $P$-value $<0.05$ ) (Figs. 2D, 3A). We performed quantitative RT-qPCR and immunofluorescence staining to confirm that the transcripts identified as differentially expressed by RNA-seq indeed showed significant enrichment in the pancreatic cell lineage (Fig. 3B,C; Supplemental Fig. 5). In Drosophila as well as some mammalian tissues, the PCP proteins are initially enriched in the apical cell membrane prior to their asymmetric distribution at either the proximal or distal side of the cell (Vichas and Zallen 2011; Wallingford 2012). Their distribution within the plane of the foregut epithelium has not been previously reported. We found Fzd2 receptor, Ror2 coreceptor, and PCP proteins such as Celsr2 and Fat1 to be enriched at the cell surface of both foregut epithelial and pancreatic progenitor cells with no obvious asymmetric distribution but completely absent in hepatic progenitors (Fig. 3C; Supplemental Fig. 5A).

In several systems, there is direct evidence that noncanonical Wnt signaling regulates cell adhesion and cytoskeleton components (Karner et al. 2009). Accordingly, in our data set, several adhesion molecules-including Claudin 4, laminin, integrin a3 (Itga3), fibronectin leucine-rich transmembrane protein 2 (Flrt2), and Flrt3-as well as cytoskeleton components such as cytokeratins CK19 and CK7 and Shroom3 showed similar differential expression, and were down-regulated in hepatocyte progenitors after lineage divergence (Fig. 3B,C; Supplemental Fig. 5B). Taken together, these results indicate that active remodeling of the foregut epithelium accompanies cell fate determination between liver and pancreas, resulting in differences in the relative adhesiveness of the cells. Moreover, our findings suggest that the noncanonical Wnt signaling might influence this cell fate decision within the foregut endoderm.

One prediction from this hypothesis is that upon exposure to noncanonical Wnt, endodermal cells acquire a pancreatic-specific differentiation program and fail to induce hepatic genes expression. To test this prediction, we first used one of the most intensively studied 

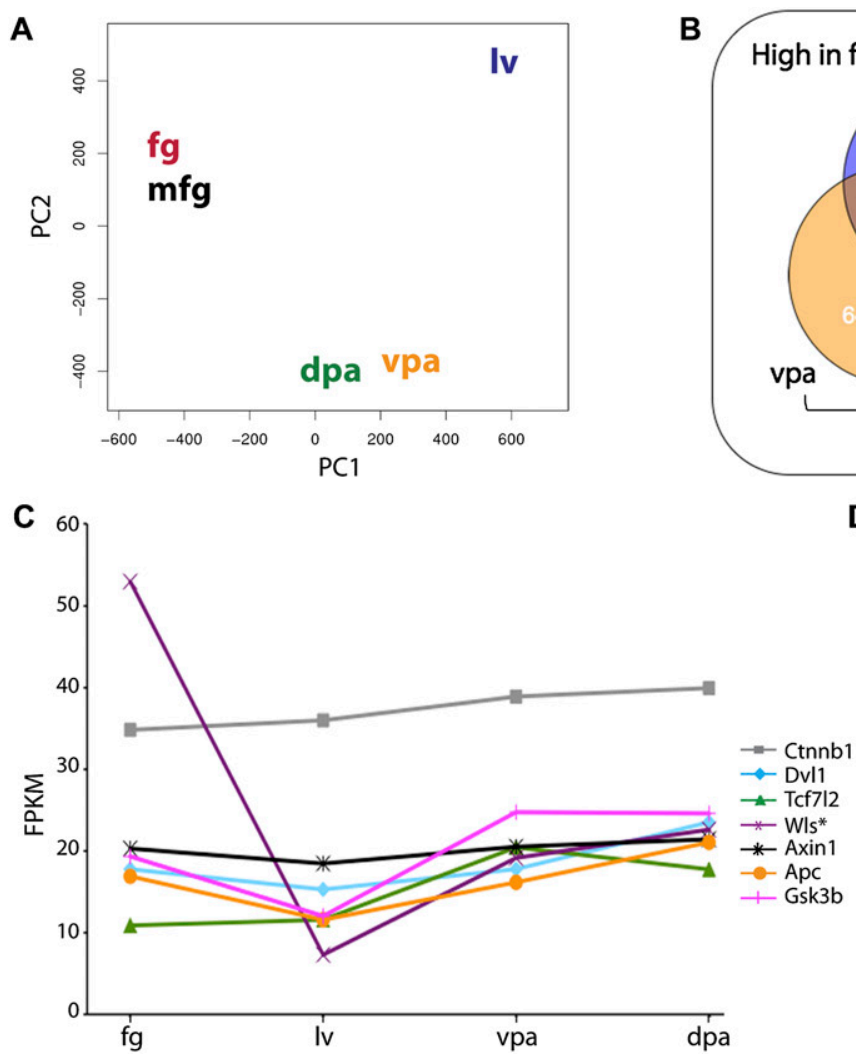
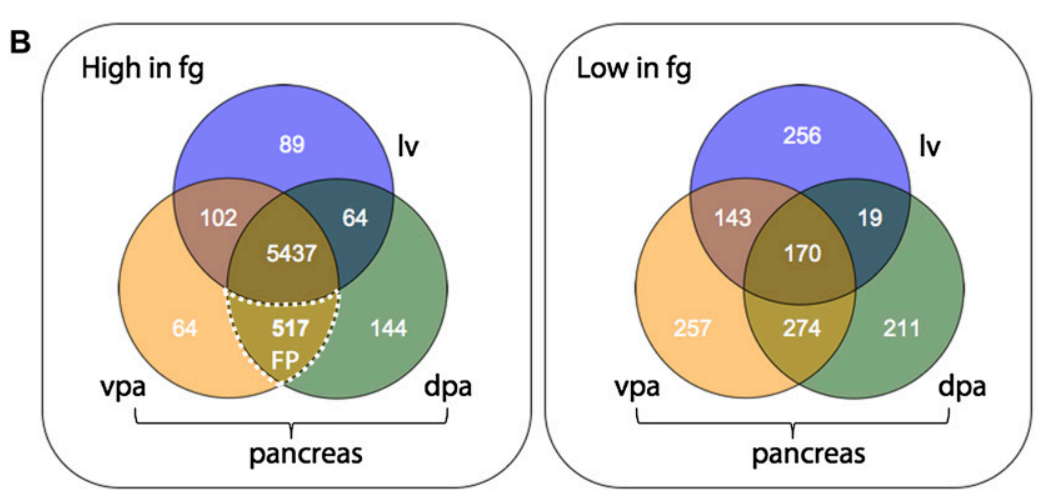

D

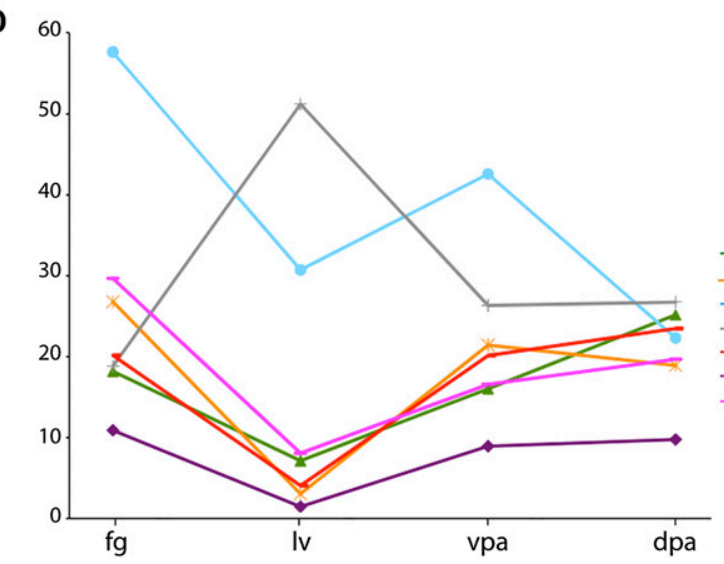

Figure 2. Temporal and spatial integration analysis of the RNA-seq-derived transcriptome profiles. $(A)$ PCA shows that the RNA-seqderived transcriptome profiles are characteristic of different progenitor cell types (for detailed description, see the Materials and Methods). (B) Venn diagrams showing the number of unique and common highly expressed transcripts between progenitor cells at different developmental stages. To further focus our analysis on subsets of genes with distinct expression patterns, we divided the working data set into two groups based on a cutoff for high expression (defined as FPKM =10, at approximately the 50th percentile of RNA-seq expression for each sample). Of 14,053 genes, 8110 could be categorized in the defined Venn regions. For example, 5437 genes exhibited relative abundance values of $>10$ FPKM in all samples, while 517 genes were highly expressed in the foregut, vpa, and dpa but not in the liver (referred to as group FP). In contrast, 89 genes were highly expressed in the foregut and liver but not in the vpa and dpa. Three-hundred-sixty-three transcripts were present (>10 FPKM) only in the fg but not in the liver, dpa, and vpa (not shown in the diagram). As shown in the PCA plot in $A$, fg and mfg were highly similar; therefore, these samples were combined together as "foregut" to simplify the visualization. $(C, D)$ Levels of Wnt signaling pathway gene expression across the fg, liver (lv), vpa, and dpa progenitors. FPKM values ( $Y$-axis) were plotted against the different progenitors cell types ( $X$-axis). $\left(^{*}\right)$ Wnt factors present in the $150 \mathrm{FP}$ group transcripts that showed significant differential regulation between the pancreas and liver.

noncanonical Wnts, Wnt5a, which we also found to be cell-autonomously produced by the E8.5 foregut endoderm (Supplemental Table 1; Supplemental Fig. 4A-C). Subsequently, at E10.5, Wnt5a expression was maintained at lower levels in pancreatic progenitor cells and was absent in hepatoblasts (Supplemental Fig. 4B,C). In addition to the endodermal expression, we found $W n t 5 a$ to be abundant in the surrounding mesenchyme at both E8.5 and E10.5, suggesting potential autocrine and/or paracrine ligand activities (Supplemental Fig. 4C). The other noncanonical Wnts, Wnt $5 b$ and Wnt $7 b$, were found to be abundant in pancreatic progenitor cells and not in hepatoblasts starting from E10.5, while they were expressed at low levels in foregut cells (Supplemental Fig. 4A,B).

Perturbations of Wnt5a signaling in Xenopus, zebrafish, and mice strongly suggest that Wnt5a activates a conserved pathway that controls cell fate, movements, and polarity during development (Moon et al. 1993; Ho et al. 2012). Moreover, the expression pattern appears conserved across species because Wnt5a is expressed in endodermal as well as surrounding mesodermal cells in the Xenopus embryo at the time of foregut organogenesis (Supplemental Fig. 4D; Zhang et al. 2013). Therefore, we first used the Xenopus laevis embryo as a model system and exposed anterior endodermal cells, which are fated to become either liver or pancreas, to soluble Wnt5a protein. In addition to the expected morphogenetic defects, Wnt5a induced pancreatic progenitor gene expression, including $P d \times 1$ and Ptf1a, and, to a lesser extent, pancreatic differentiation markers, such as Glucagon $(\mathrm{Gcg})$ and Trypsin (Fig. 4A,B). Concomitantly, Wnt5a treatment repressed expression of the hepatic genes Hex, For1, and Albumin in the same endodermal cells without affecting other endodermal cell fates, as judged by unchanged Nkx6.2 (duodenum) and Sox2 (stomach) expression levels (Fig. 4B; Chalmers et al. 2000; Dichmann and Harland 
A

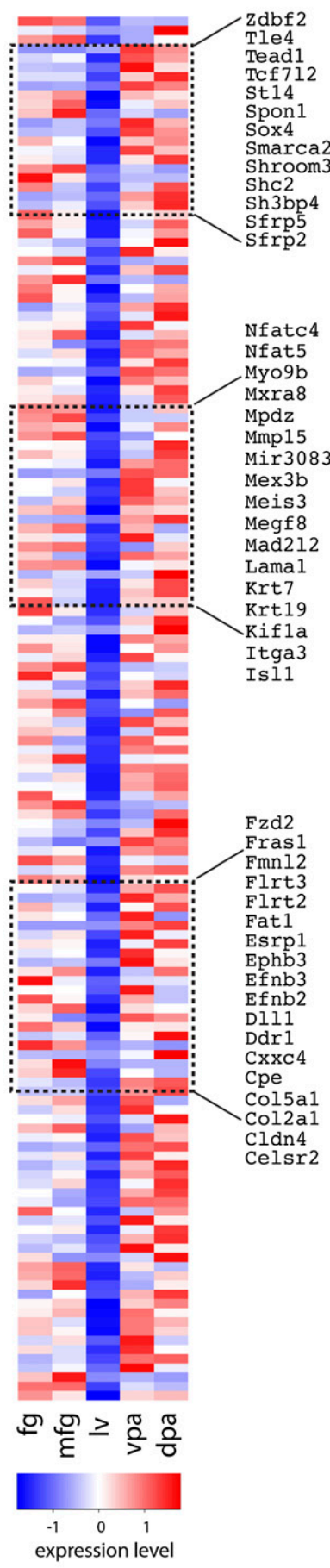

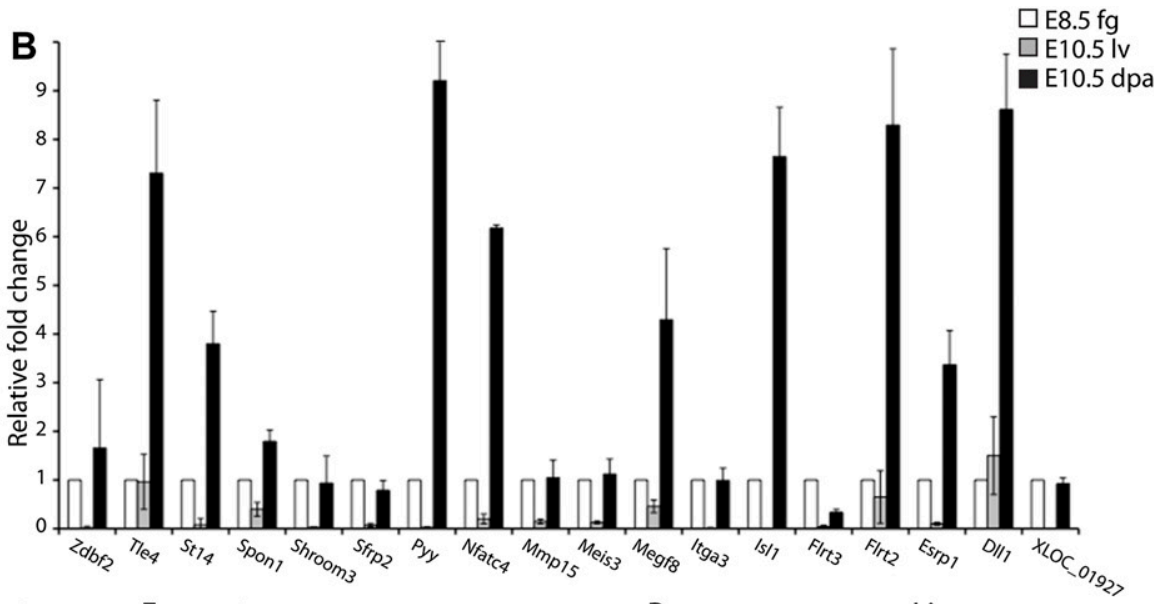

C
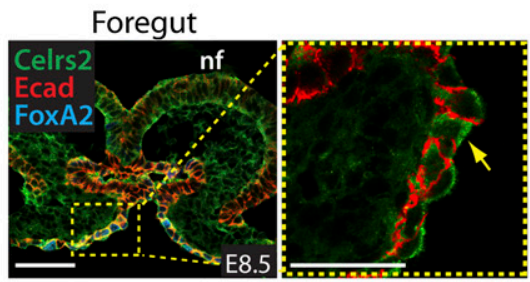

Pancreas
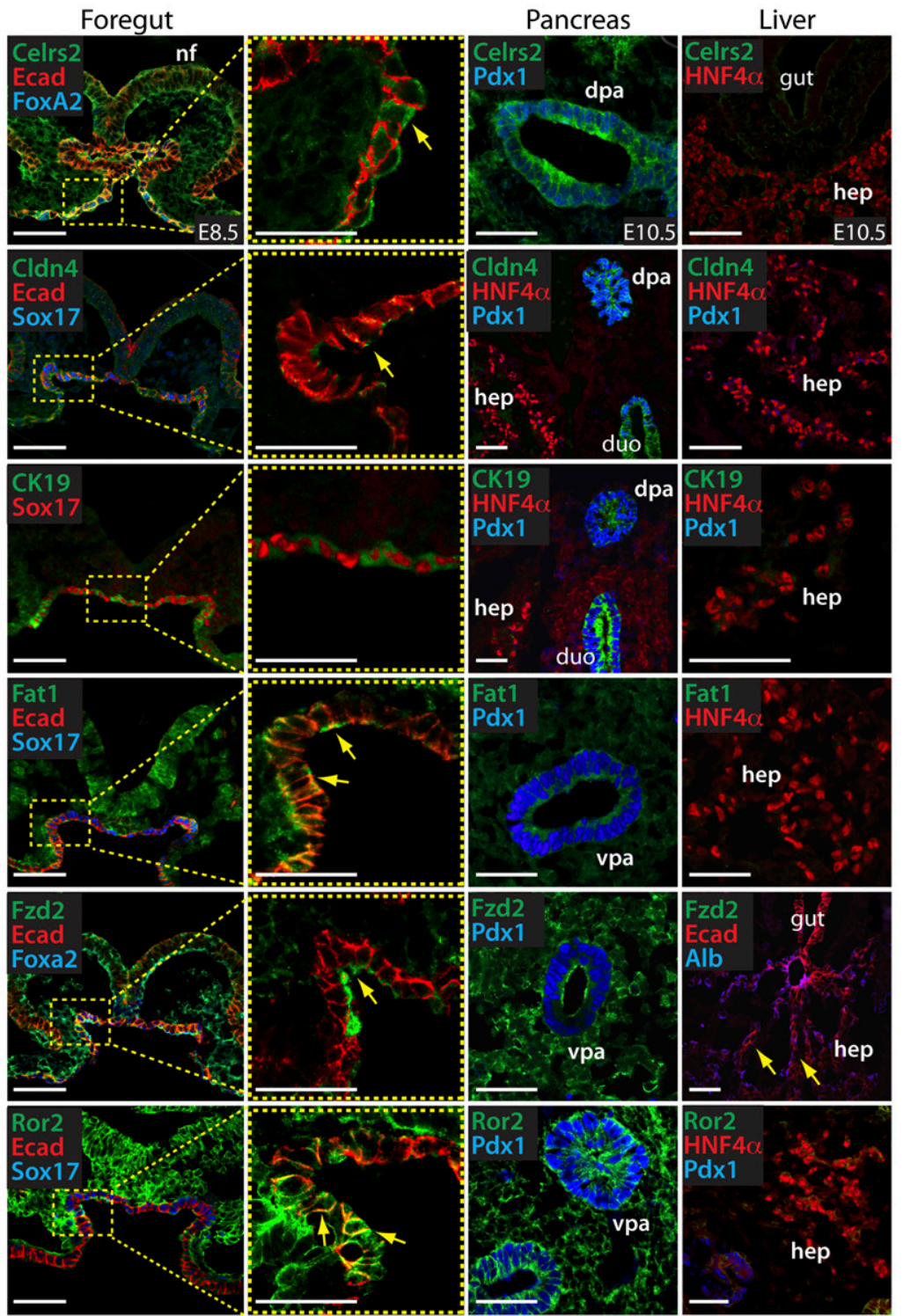

Figure 3. Analysis of candidate regulators of the pancreatic versus hepatic fate decision. $(A)$ Heat map view of the FP group transcripts that were differentially expressed between any of two samples (150 transcripts out of 517; Cufflinks, $P$-value $<0.05)$. Colors represent high (red) or low (blue) expression values based on $Z$-score normalized FPKM values for each gene. White represents the average between red (high) and blue (low) expression values. Dashed boxes highlight gene sets validated by either RT-qPCR or immunofluorescence analyses. $(B)$ RT-qPCR validation of a subset of differentially expressed genes of the FP group. Data were normalized to that of succinate dehydrogenase (SDHA) and are represented as fold change compared with the E8.5 foregut sample (set to 1 as calibrator). Error bars represent \pm SEM. (C) Immunofluorescence analysis validated the exclusive localization of Celrs2, Claudin 4 (Cldn4), CK19, Fat1, Fzd2, and Ror2 in E8.5 foregut endoderm (see arrows in the insets) and pancreatic progenitors and their absence in the liver (see arrows). Micrographs show cross-sections of E8.5 and E10.5 mouse embryos. Bars, $50 \mu \mathrm{m}$. (duo) Duodenum; (hep) hepatic progenitors; (lv) liver; (nf) neural folds. 


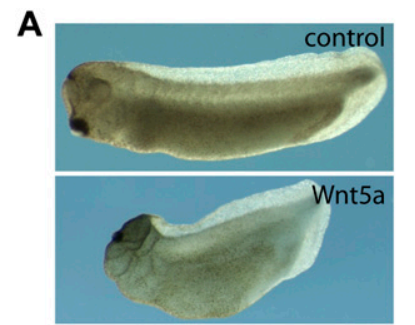

C
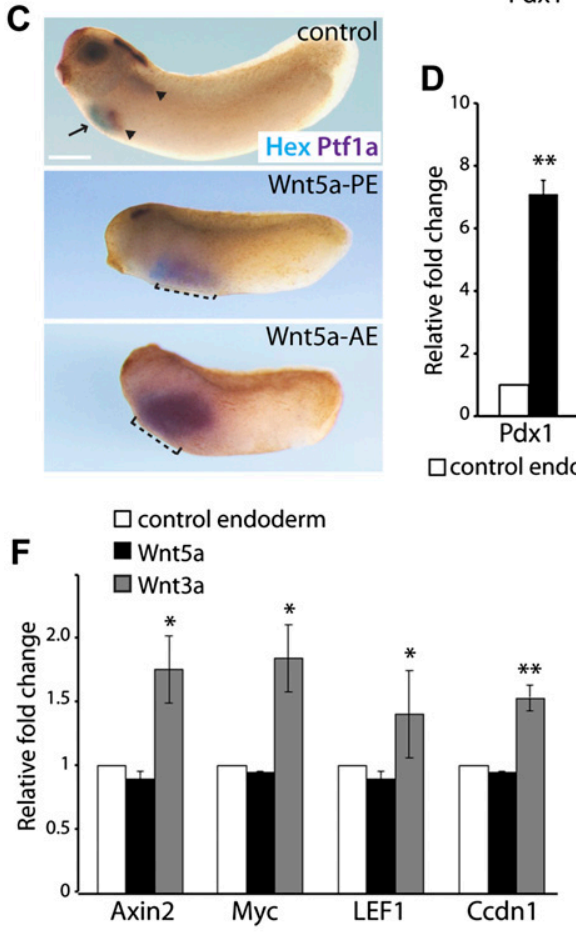

D
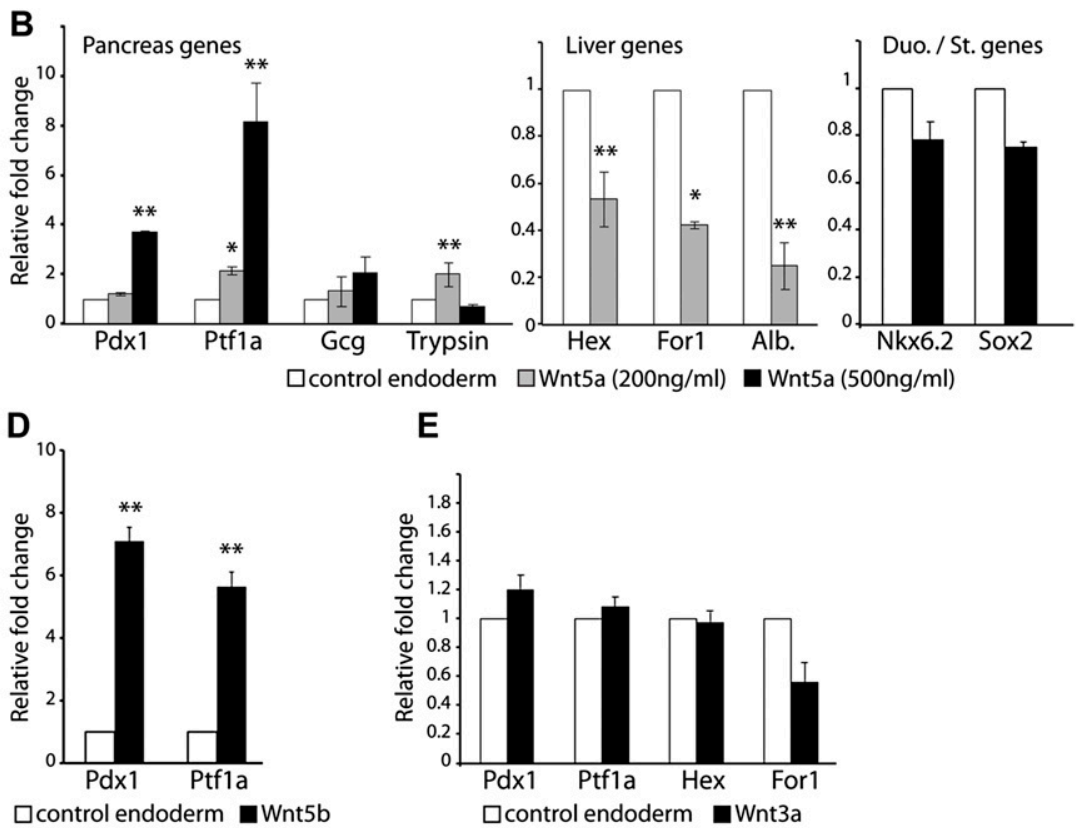

E

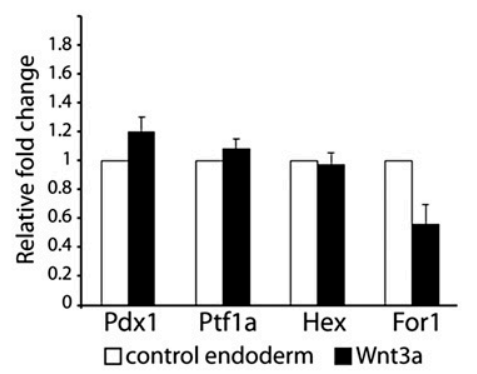

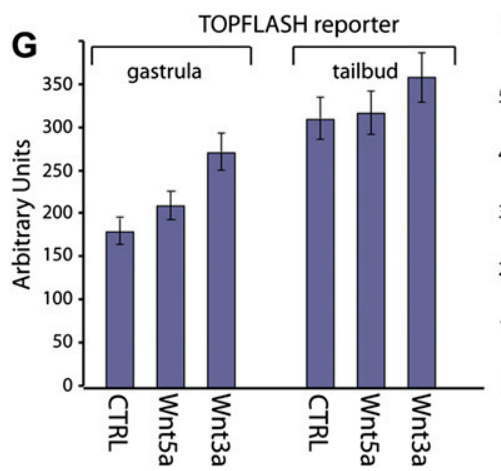
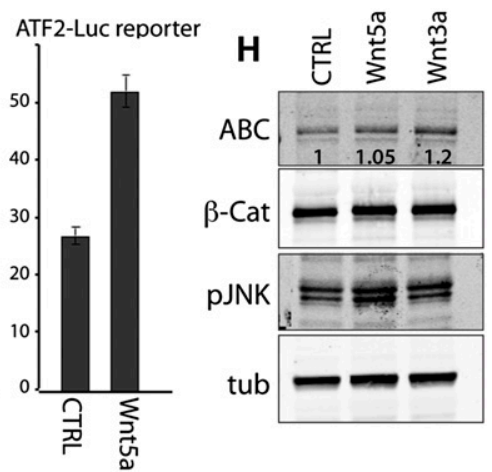

Figure 4. Noncanonical Wnt5a activity promotes pancreatic versus hepatic fate in the anterior endoderm. $(A)$ Xenopus embryos injected with Wnt5a mRNA showed a shortened and mildly bent body at the tailbud stage, as previously described (Kim et al. 2005). (B) Endodermal explants were cultured in the presence of Wnt5a recombinant protein from stage 10, collected at the tadpole stage, and assayed for expression of the indicated pancreatic, hepatic, and duodenum/stomach genes by RT-qPCR analysis. Untreated anterior endodermal explants were used as control. Data were normalized to that of ornithine decarboxylase (ODC) and are represented as fold changes compared with untreated endoderm sample (set to 1 as calibrator). Error bars represent \pm SEM. (C) Whole-mount double in situ hybridization analysis of Hex (light blue) and Ptf1a (purple) in control and Wnt5a-injected Xenopus embryos at the tadpole stage. The arrow indicates Hex expression in the liver bud, and arrowheads indicate Ptf1a expression in the two pancreatic buds (dorsal and ventral buds). Dashed lines mark expanded Ptf1a expression in the injected embryos. Total number of injected embryos $=61 ; 41 \%$ showed visible expansion of Ptf1a. (AE) Anterior endoderm; (PE) posterior endoderm. (D) RT-qPCR analysis of endodermal explants treated with Wnt5b $(200 \mathrm{ng} / \mathrm{mL})$ recombinant protein. Data were normalized to that of ODC and are represented as fold changes compared with untreated endoderm sample (set to 1 as calibrator). (E) RT-qPCR analysis of endodermal explants treated with $500 \mathrm{ng} / \mathrm{mL}$ Wnt3a recombinant protein. Data were normalized to that of ODC and are represented as fold changes compared with untreated endoderm sample (set to 1 as calibrator). $(F)$ RT-qPCR analysis of direct downstream target genes of the Wnt/ $\beta$-catenin pathway in endodermal explants treated with $500 \mathrm{ng} / \mathrm{mL}$ Wnt5a or $500 \mathrm{ng} / \mathrm{mLWnt} 3$ a recombinant protein. Data were normalized to that of $O D C$ and are represented as fold changes compared with untreated endoderm sample (set to 1 as calibrator). (G) TOPFLASH and ATF2-luc reporter assays in Xenopus embryos. Four-cell stage embryos were injected into the vegetal blastomeres with 50 pg of TOPFLASH or 100 pg of ATF2-luc plus 25 pg of Renilla luciferase reporter plasmids. Endodermal explants were dissected at stage 9 and either left untreated as control (CTRL) or exposed to $500 \mathrm{ng} / \mathrm{mL} \mathrm{Wnt5a} \mathrm{or} 500 \mathrm{ng} / \mathrm{mL}$ Wnt3a recombinant protein, as indicated. Luciferase reporter assays were carried out in explants lysed at gastrula and early tailbud stages. $(H)$ Western blot analysis of dissected anterior endodermal explants either left untreated as control (CTRL) or exposed to Wnt5a or Wnt3a recombinant protein. The relative ABC/tubulin levels in the treated explants compared with the control, which was set to 1.0 , are indicated. $\left(\beta\right.$-cat) Total $\beta$-catenin; (tub) $\alpha$-tubulin. $\left({ }^{\star}\right) P<0.05$; $\left(^{\star \star}\right) P<0.01$, as determined by the REST program statistical analysis (Pfaffl et al. 2002).

2011). Similar effects were observed when Wnt5a mRNA was microinjected into either anterior or posterior endodermal cells of the Xenopus embryo, resulting in ex- panded Ptf1a expression beyond its normal boundaries (Fig. 4C). Furthermore, we found that activation of the noncanonical Wnt pathway using a different noncanonical 
ligand, the Wnt5b recombinant protein, also induced a strong expression of pancreatic progenitor transcription factors in anterior endodermal cells (Fig. 4D). Finally, we examined whether the exposure to the noncanonical Wnt pathway has an effect on cell proliferation in the anterior endoderm. Immunostaining of phospho-histone $\mathrm{H} 3$ (PHH3) to mark cells undergoing mitosis revealed no significant differences in proliferating cell number in the foregut between Wnt5a-injected and uninjected early tadpole stage embryos (Supplemental Fig. 4E).

On the other hand, the establishment of proper levels of canonical Wnt/ $\beta$-catenin signaling and its temporal sequential activation in the anterior endoderm (Fig. 4) are known to be essential for foregut identity and organ formation (Ober et al. 2006; Li et al. 2008; Angers and Moon 2009; Puri and Hebrok 2010; Zhang et al. 2013). After adding Wnt3a recombinant protein, a well-known canonical Wnt activator (Grigoryan et al. 2008; Clevers and Nusse 2012), to anterior endodermal cells, we observed that the levels of expression of hepatic and pancreatic genes were unchanged when compared with control endoderm, ruling out an apparent choice of cell type between liver or pancreas (Fig. 4E).

The noncanonical Wnt signaling is able to antagonize the canonical signaling in certain biological contexts (Grumolato et al. 2010; Ho et al. 2012). To distinguish between these two possible Wnt5a activities (e.g., noncanonical Wnt activity or antagonist effect on Wnt/ $\beta$-catenin) in the context of liver and pancreas lineage divergence, we performed a series of quantitative assays and biochemical analysis in the Xenopus endoderm. First, we analyzed the transcriptional responses to both pathways in endoderm cells treated with Wnt5a by (1) examining direct downstream target genes of Wnt/ $\beta$-catenin pathway and (2) using specific luciferase reporter assays based on TCF/LEF (TOPFLASH) and ATF2 response elements for canonical and noncanonical Wnt, respectively (Ohkawara and Niehrs 2011; Clevers and Nusse 2012). RT-qPCR analysis showed that Wnt3a treatment increased the expression levels of direct Wnt target genes such as Cyclin-D1 (Ccdn1), Lef-1, Myc, and Axin 2, whereas Wnt5a treatment does not affect their expression in anterior endodermal cells (Fig. 4F). This is in line with the RNA-seq profiles of mammalian pancreatic and hepatic progenitor cells displaying comparable expression levels of these downstream target genes (Fig. 2; Supplemental Table 2). Furthermore, we found that exposure to Wnt5a does not suppress endogenous canonical Wnt activity in the endoderm, as monitored by the Wnt/ $\beta$-catenin TOPFLASH reporter assay, whereas it induces the noncanonical Wnt ATF2 reporter activity (Fig. 4G). Notably, endogenous canonical Wnt/ $\beta$-catenin transcriptional activity accumulates in the Xenopus endoderm from gastrulation onward (Fig. 4G; Angers and Moon 2009; Ohkawara and Niehrs 2011). This results in elevated basal levels of TOPFLASH luciferase activity in the untreated endodermal cells (control [CTRL]) and is consistent with the mild induction of the TOPFLASH reporter observed upon exposure to Wnt3a-soluble protein (Fig. 4G).
Next, we conducted biochemical assays on endoderm explants treated with Wnt5a and Wnt3a to analyze the status of activation of $\beta$-catenin signaling. Western blot analysis showed that levels of total and active $\beta$-catenin (ABC) protein (dephosphorylated on Ser37 and Thr41) (van Noort et al. 2002) remained unchanged in Wnt5atreated anterior endodermal cells when compared with control endoderm (Fig. 4H), whereas Wnt3a moderately increased dephosphorylated $\beta$-catenin levels. As expected, Wnt5a induced JNK phosphorylation, reflecting noncanonical Wnt activation (Fig. 4H). Taken together, these results rule out Wnt5a function as an antagonist of the canonical Wnt pathway and support a role of the noncanonical Wnt signaling in the pancreatic versus hepatic fate decision in a $\beta$-catenin-independent manner.

Last, we sought to expand the functional analysis on the noncanonical Wnt pathway directly to mammalian endodermal cells. First, we used the mouse embryonic stem cell (mESC) system to model ex vivo endoderm development (Rossant 2011). In particular, to induce pancreatic specification, mESCs were stimulated using a step-wise protocol in a monolayer culture adapted from previously published studies (D'Amour et al. 2006; Nostro et al. 2011; Wang et al. 2011; Chen et al. 2013). RT-qPCR analysis revealed first up-regulation of definitive endoderm transcription factors such as Sox17 and Foxa2 followed by sequential induction of pancreatic progenitor genes, including $P d x 1$, Sox9, Pax6, and Islet 1 (Isl1) (Fig. 5A). Consistent with pancreatic differentiation, the levels of expression of Sox9, Pax6, and Is 11 increased with time and upon exposure to additional cytokines (e.g., noggin, retinoic acid, and cyclopamine), while no or minimal induction of the hepatic marker Albumin was detected (Fig. 5A). Most differentiation protocols use FGF10 to pattern the definitive endoderm-induced population toward the pancreatic endoderm fate within the foregut and the subsequent pancreatic progenitor fate (D'Amour et al. 2006; Nostro et al. 2011). To determine whether the noncanonical Wnt pathway would enhance pancreatic specification, we examined the consequences of treating definitive endoderm cultures generated from ESCs with Wnt5a in the presence or absence of FGF10. Interestingly, Wnt5a enhanced the levels of $P d x 1$ expression compared with the standard pancreatic endoderm differentiation conditions (Fig. 5B). In addition, the positive effect of Wnt5a was even more evident in definitive endoderm cells cultured in the absence of FGF10, as its addition restored the induction of $P d x 1$ expression to pancreatic endoderm standard levels (Fig. 5C). Importantly, the duodenal marker $C d x 2$ was not induced in pancreatic endoderm cultures differentiated in the presence of Wnt5a, and the slight induction of Albumin expression in pancreatic endoderm cultures was downregulated by Wnt5a treatment, supporting a specific effect on pancreatic fate (Fig. 5B). In addition, we found that other pancreatic progenitor transcription factors, including $N k x 6.1$ (at the pancreatic endoderm stage), Sox9, and Pax6 (at the pancreatic progenitor stage), were strongly induced in both the absence and presence of Wnt5a, although a further enhancement of their transcript levels 
Rodríguez-Seguel et al.

A do

d2

d5

ESC DE PE PP

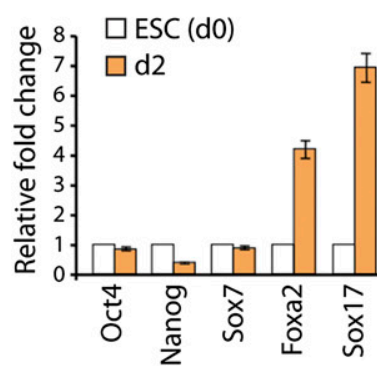

B

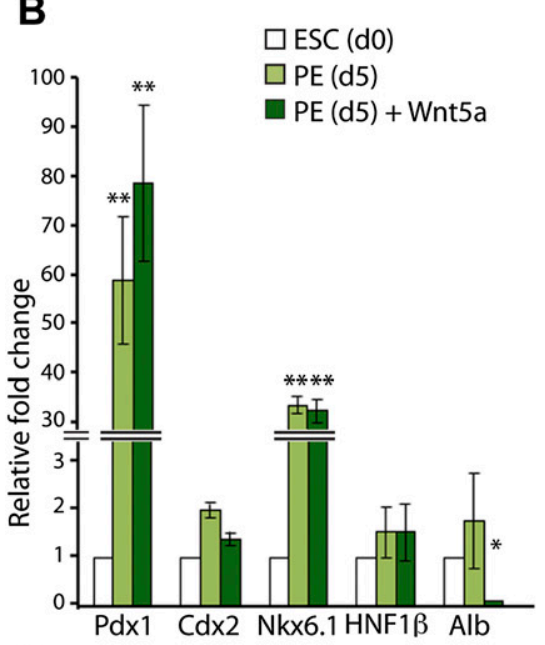

D

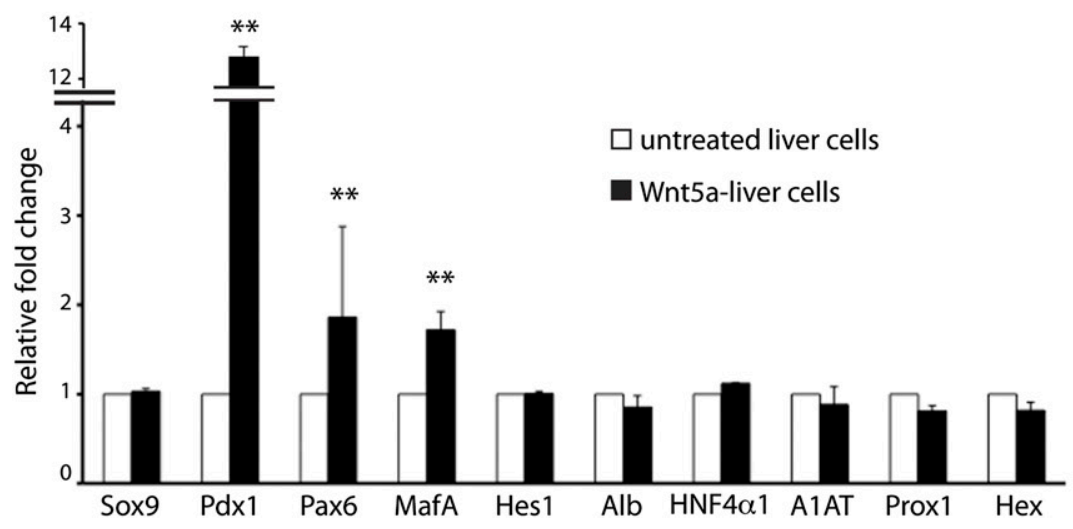

C
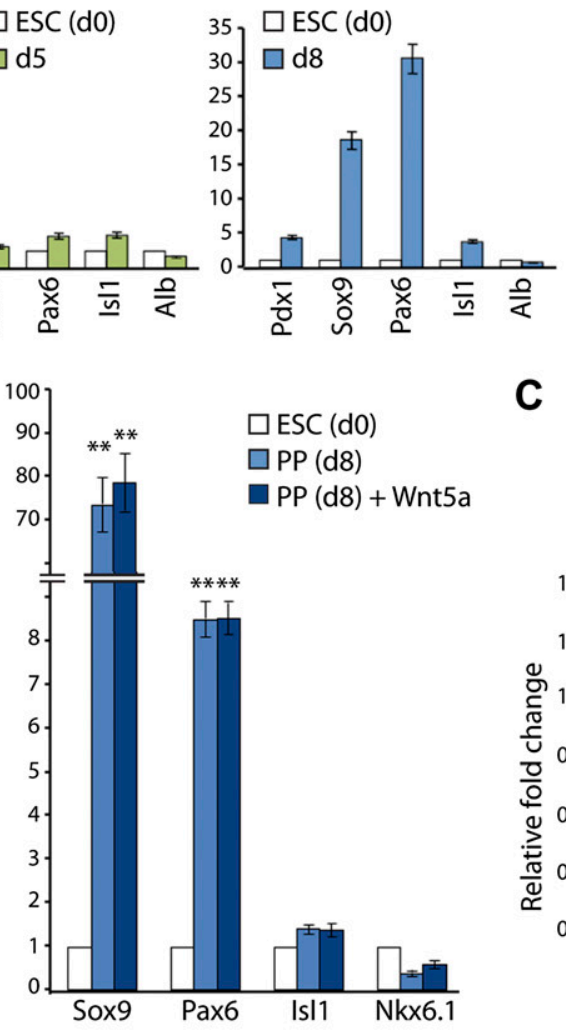

$\square P E(d 5)$

$\square$ PE - FGF10

$\square \mathrm{PE}+\mathrm{Wnt} 5 \mathrm{a}$

$\square$ PE - FGF10 + Wnt5a

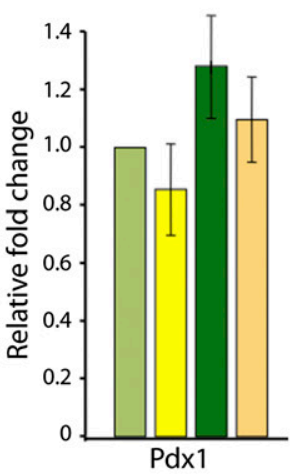

Figure 5. Conserved Wnt5a activity in promoting pancreatic fate. (A) Directed differentiation of mESC monolayer cultures into pancreatic progenitors. RT-qPCR analysis evaluating definitive endoderm (DE), pancreatic endoderm (PE), and pancreatic progenitor (PP) gene expression at different stages of differentiation. Untreated mESCs were used as control (d0). Data were normalized to that of SDHA and are represented as fold changes compared with control (d0) mESCs (set to 1 as calibrator). (B) Day 5 and day 8 mESC cultures were analyzed by RT-qPCR for the expression of the indicated genes following either standard pancreatic endoderm and pancreatic progenitor culture conditions or in the presence of Wnt5a recombinant protein (PE + Wnt5a and PP + Wnt5a). RT-qPCR data were normalized to that of SDHA and are represented as fold changes compared with control (d0) mESCs (set to 1 as calibrator). Error bars represent \pm SEM. $(C)$ Day 5 mESC cultures were analyzed by RT-qPCR for the expression of the pancreatic gene Pdx1 following standard pancreatic endoderm culture conditions in the absence of FGF10 (PE - FGF10), the presence of Wnt5a (PE + Wnt5a), or the presence of Wnt5a but without FGF10 (PE - FGF10 + Wnt5a). RT-qPCR data were normalized to that of SDHA and are represented as fold changes compared with standard pancreatic endoderm condition (set to 1 as calibrator). Error bars represent \pm SEM. (D) BAML liver cells cultured in the presence of $200 \mathrm{ng} / \mathrm{mL} \mathrm{Wnt5a}$ for $2 \mathrm{wk}$ were assayed for expression of the indicated pancreatic and hepatic genes by RT-qPCR analysis. Untreated BMAL liver cells were used as control. Data were normalized to that of SDHA and are represented as fold changes compared with untreated liver cells (set to 1 as calibrator). Error bars represent \pm SEM. $\left(^{\star}\right) P<0.05 ;\left({ }^{\star \star}\right) P<0.01$, as determined by the REST program statistical analysis (Pfaffl et al. 2002). (Alb) Albumin. 
(as seen for $P d \times 1$ ) was not detectable in these culture conditions (Fig. 5B). This finding suggests that patterning of anterior endoderm with noncanonical Wnt signaling might be important for optimal pancreatic specification in protocols for directed differentiation of ESCs.

Next, we asked whether modulation of the noncanonical Wnt pathway might promote the pancreatic program in differentiated liver cells. To this aim, we used a mammalian ex vivo model system, the nontransformed BAML hepatic cell line (Fougère-Deschatrette et al. 2006). The BAML cells were established from adult mouse livers and were shown previously to retain hepatic differentiation hallmarks and repopulate the liver in vivo (FougèreDeschatrette et al. 2006). We cultured the liver cells in their standard hepatocyte culture medium in the presence or absence of Wnt5a. After prolonged exposure to Wnt5a, we observed significant induction of the pancreatic genes, including $P d \times 1, P a \times 6$, and $M a f A$, in liver cells, whereas the level of expression of the liver markers Albumin and a1-antitrypsin (A1AT) and the liver-specific transcription factor $H N F 4 \alpha 1$ was not affected (Fig. 5C). This suggests that Wnt5a might also facilitate fate conversion of liver cells into pancreatic fate but does not itself possess the ability to suppress hepatic identity.

All together, these results implicate a novel and conserved role for the noncanonical Wnt signaling pathway in promoting the pancreas versus liver fate decision in the endoderm. In particular, Wnt5a appears to exert specific regulation on $P d \times 1$ gene expression, which is conserved in both the mouse and Xenopus endoderm (Figs. 4, 5). An entire set of noncanonical Wnt transducers was identified in our analysis (Figs. 2, 3); whether Wnt5a and Wnt5b activities in this cell lineage decision are due to signaling through components of the PCP, Ror2/JNK, or Calcium/ NFAT pathways (Kim et al. 2005; Ober et al. 2006; Li et al. 2008; Puri and Hebrok 2010) deserves further investigation.

\section{Spatially distinct expression patterns mark the progenitor populations}

Our data provide an opportunity to systematically examine additional in vivo expression patterns within the hepatic and pancreatic endoderm. For example, we focused on identifying transcriptional differences between discrete domains of the foregut endoderm at E8.5 (fg vs. $\mathrm{mfg}$ ), before organ rudiments were formed (Fig. 6). Only 21 transcripts displayed statistically significant differential expression between these two regions (Fig. 6A). The small number of differentially expressed transcripts might reflect the active movements and intermingling of cells across the foregut at this stage (Tremblay and Zaret 2005). Among them were only a few genes that had been previously reported in the endoderm, including Pyy, Otx2, and Sox2 (Hou et al. 2007), but the majority was not known to be expressed in this territory (Fig. 6A-D). The expression of some of these foregut genes was maintained in either liver progenitors (E2f2), ventral pancreatic progenitors (Celsr2 and Nr1h5), or both (Cdkn1c), representing interesting candidates for lineage tracing studies to establish the exact contribution of progenitor domains within the endoderm.

Notably, in the ventral foregut epithelium, we detected Hox genes encoded by the HoxD clusters (Hoxd3 and Hoxd4) as well as a spliced noncoding RNA that is transcribed antisense to the Hoxb5 and Hoxb6 genes (0610040B09Rik), which might be relevant for their regulation (Fig. 6A; Supplemental Fig. 6; Dinger et al. 2008). A clear spatial difference in the expression of $H o x$ genes was evident also at later stages in the pairwise comparison between E10.5 vpa and dpa data sets (Supplemental Fig. 7). Hox genes such as Hoxa3, Hoxa4, Hoxa7, Hoxb3, Hoxb6, Hoxb7, and Hoxb8 were abundantly expressed in the dorsal pancreatic region but were absent or at undetectable levels in the vpa and liver (Fig. 6A; Supplemental Fig. 7). Using RT-qPCR and in situ hybridization, we confirmed the differential expression between the dpa and vpa of selected Hox genes, including Hoxa7, Hoxb6, and Hoxb8 (Fig. 6F-I). Notably, Hoxa 7 and Hoxb6 seem to be expressed in a heterogenous manner in the dpa, possibly marking specific cell types (Fig. 6F,G). Taken together, our findings suggest that a specific Hox code exists in the developing foregut as well as within the pancreatic territory, and its biological significance needs to be assessed (Grapin-Botton and Melton 2000; Iimura et al. 2009).

Besides the Hox genes, we detected large differences in gene expression between the ventral and dorsal pancreatic buds at the onset of pancreas organogenesis (192 transcripts; absolute value of $\log 2$ fold change $>3, P$-value $<$ 0.05) (Supplemental Fig. 7). To determine whether there was any recognizable biological relevance to the expression patterns, we analyzed GO terms with respect to molecular functions and found significantly enriched annotations in each cluster. Strong association with extracellular binding activities (GO: 0005488; 62 genes; $P$-value $<0.000129$ ) was found in the vpa cluster, whereas the dpa cluster showed strong enrichment for DNA-binding transcription factor function (GO: 0003700; 25 genes; $P$-value < $\left.9.41 \times 10^{-18}\right)$.

Our comprehensive analysis further illustrates how different the dorsal and ventral pancreatic progenitors are from each other. Despite these differences at E10.5, both pancreatic rudiments give rise to endocrine and exocrine cells at later stages and eventually fuse to form the definitive pancreas (Puri and Hebrok 2010). Our data set will enable a clear understanding of how distinct transcriptional programs might lead to the same cell type, allowing, for instance, the identification of alternative modes that can be used to program stem cells toward pancreatic endocrine fates.

\section{Concordant gene signatures between mouse and human pancreatic progenitors}

Next, we sought to evaluate the evolutionary importance of our transcriptome analysis of pancreatic progenitor cells and its relevance to human pancreas development. Given the fact that genomic analysis on equivalent developmental stage human material is not 

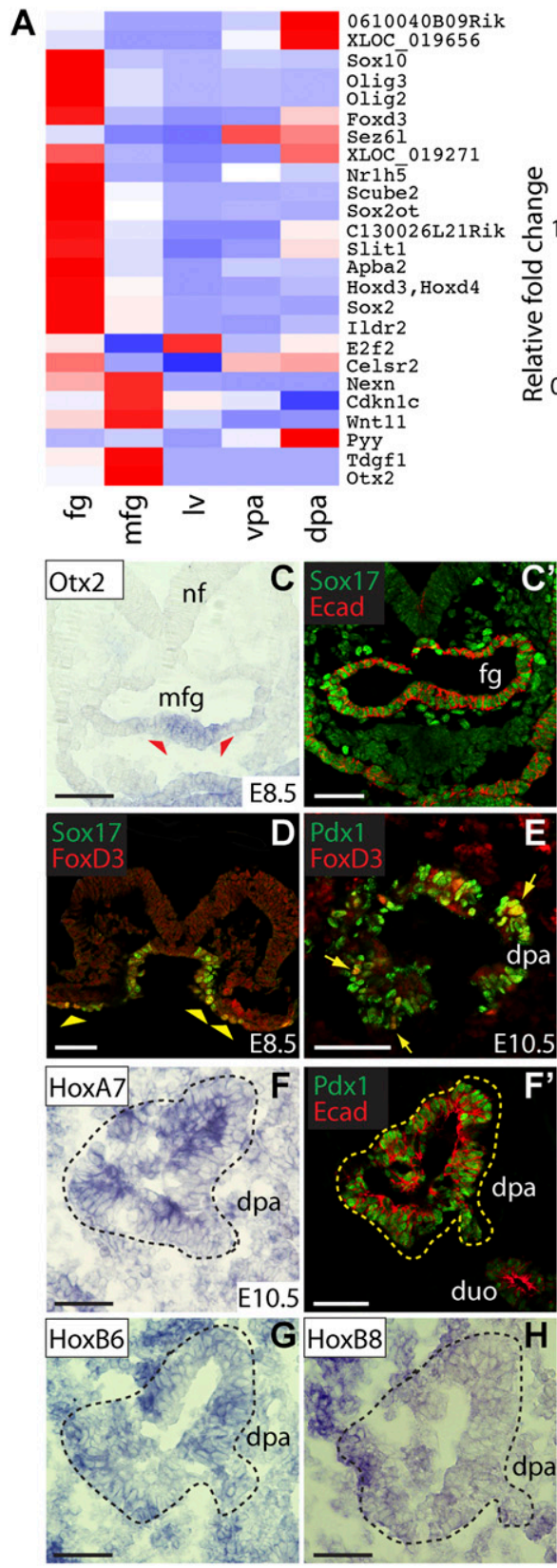

HoxB8

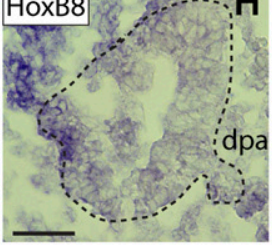

西

(1)
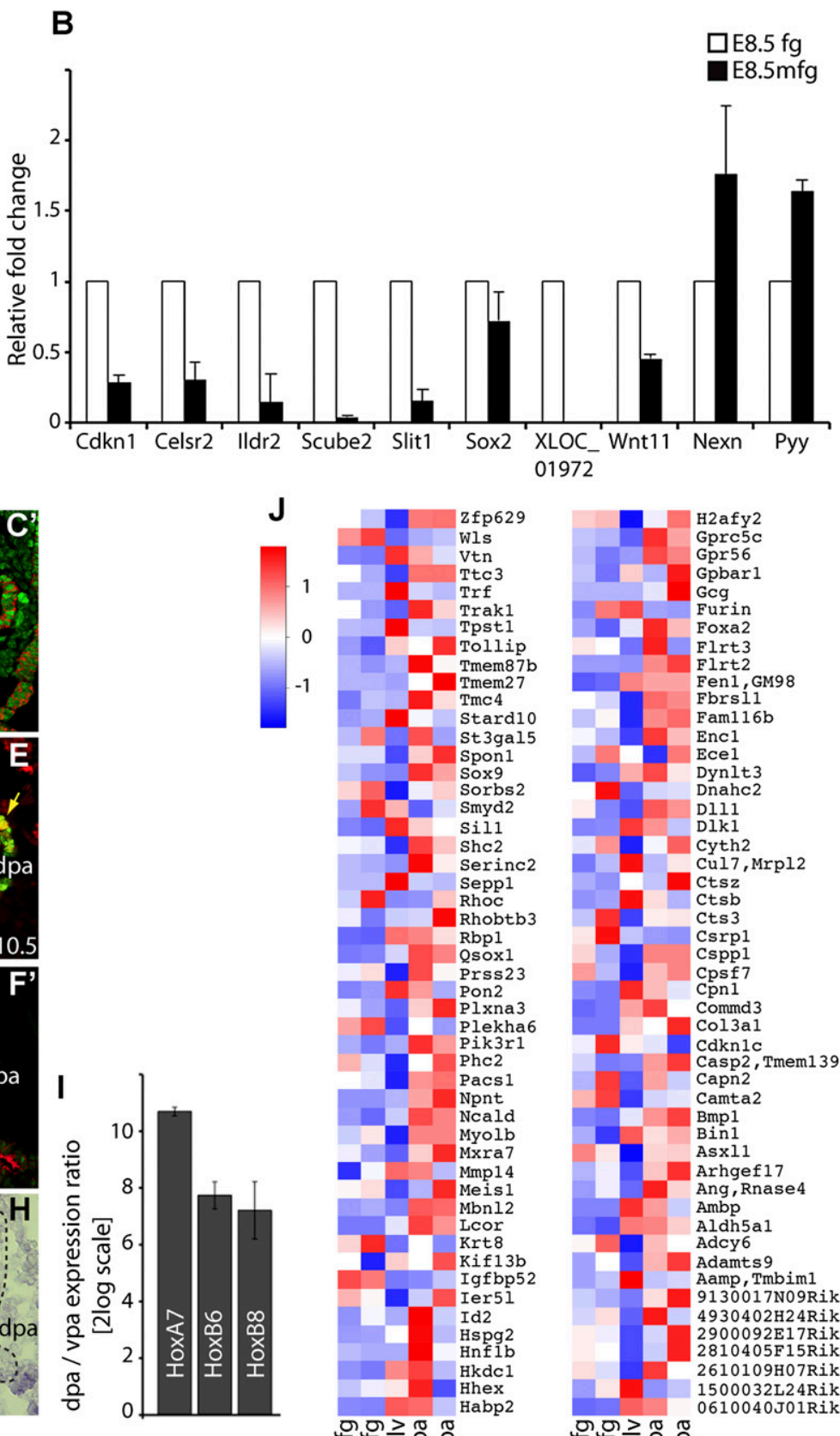

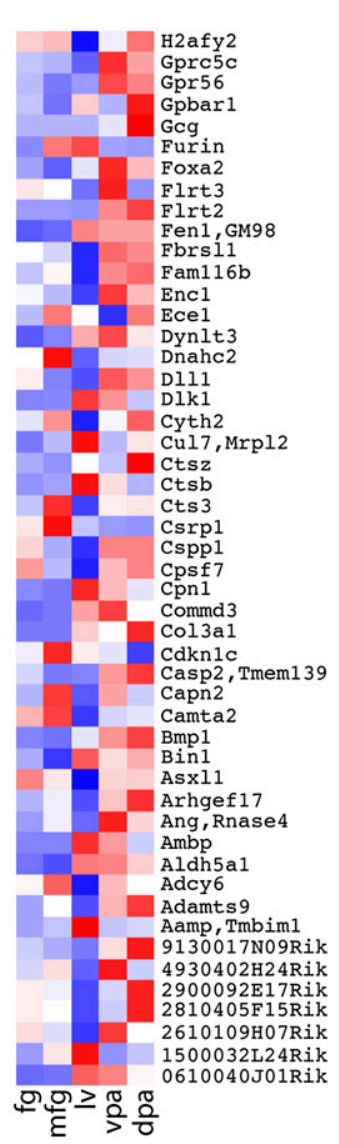

Figure 6. Identification of distinct spatial patterns within the mouse foregut endoderm. (A) Heat map view of the transcripts that showed significant differential expression between E8.5 fg and $\mathrm{mfg}$ (21 transcripts). Colors represent high (red), low (blue), or average (white) expression values based on $Z$-score-normalized FPKM values for each gene. (B) RT-qPCR validation of a subset of the foregut differentially expressed genes. The XLOC_019271 is not supported by any spliced ESTs or Genscan predictions. By exon junction analysis, we predicted a gene model and validated the expression of this novel transcript in the fg and dpa by RT-qPCR (see also Fig. 3B; Supplemental Fig. 8). Data were normalized to that of SDHA and are represented as fold change compared with the E8.5 fg sample (set to 1 as calibrator). Error bars represent \pm SEM. $(C-H)$ Immunofluorescence and in situ hybridization analyses validated the expression of the indicated genes in the E8.5 foregut endoderm (see arrowheads) and/or dorsal pancreatic rudiments (demarcated by dashed line). At E8.5, Otx2 expression was detected in the $\mathrm{mfg}(C)$, which coexpressed Sox 17 and E-cadherin (Ecad), as shown by immunofluorescence staining on serial section $\left(C^{\prime}\right)$. FoxD3 expression was detected in the E8.5 fg endoderm $(D$; see arrowheads) and dpa cells at E10.5 $(E)$. Arrows in $E$ indicate FoxD3/Pdx1-double-positive cells. At E10.5, Hox gene expression was detected in the dorsal pancreatic rudiment $(F-H)$, which coexpressed Pdx1 and E-cadherin (Ecad), as shown by immunofluorescence staining on serial section $\left(F^{\prime}\right)$. Bars, $50 \mu \mathrm{m}$. $(I)$ RT-qPCR validation of the indicated Hox genes showed differential expression between the vpa and dpa. Data were normalized to that of SDHA and are shown as expression ratio (2-log values) of dpa sample versus vpa sample. Error bars represent \pm SEM. (J) The heat map shows the expression of 100 mouse genes found to be expressed in both the vpa and dpa (>10 FPKM) and whose human orthologs are also expressed in human pancreatic "progenitor-like" cells (Micallef et al. 2012). 
RNA-seq of hepatic and pancreatic progenitor cells

available, we compared our murine RNA-seq data sets with available microarray data obtained from human ESC (hESC)-derived pancreatic-like cells (Micallef et al. 2012).

Human pancreatic progenitor state was defined by the top 400 up-regulated genes in hESC-derived pancreatic cells versus undifferentiated hESCs (Micallef et al. 2012). Importantly, we restricted our analysis only to those genes that were up-regulated in hESCs differentiated into a specific pancreatic differentiation stage that precedes the onset of overt $\beta$-cell differentiation, representing the closest gene signature of human pancreatic progenitors. This corresponds to the fraction of "FACS-sorted insulin-GFP-negative cells" obtained after application of published differentiation protocols to undifferentiated $I N S^{G F P / w}$ hESCs (Micallef et al. 2012). Mouse genes from the RNA-seq experiments were matched to their corresponding human homologs using the NCBI database HomoloGene (http://www.ncbi.nlm.nih.gov/homologene; see also the Materials and Methods). Importantly, we found that that $25 \%$ of the 400 human homolog genes are also markers of pancreas progenitors in mice, displaying high gene expression in the E10.5 vpa and dpa data sets despite the methodological and temporal differences (Fig. 6J).

\section{Discussion}

In conclusion, we devised a strategy to purify liver and pancreas progenitors directly from the endoderm of Tg(Prox1-EGFP) reporter mouse embryos. Using RNAseq, we profiled the purified progenitor cells and identified transcripts that are differentially expressed across the pancreatic and hepatic endoderm at distinct developmental stages. Importantly, we uncovered a unique noncanonical Wnt signaling signature in the emergence of pancreas versus liver from endoderm progenitors that appears conserved across phylogenetically distant species. Our results provide an invaluable resource for lineage tracing analysis to pinpoint the exact origin of the hepato-pancreatic lineage and isolation of transient progenitor cell populations. Moreover, our transcriptional data lay the foundation for further targeted functional studies of developmental regulators of the liver and pancreas fate decision that will be relevant to humans. Indeed, when we compared our RNA-seq results with those previously obtained using microarray in hESCs differentiated into pancreatic-like progenitors (Micallef et al. 2012), we identified concordant gene signatures (e.g., similar markers of pancreatic progenitors: Wls, FLRTs, and Meis). These results suggest direct implications of our findings in developing novel strategies to generate pancreas progenitors and $\beta$ cells for clinical transplantation from cellular programming of stem cells or induced pluripotent stem cells (iPSCs) and from reprogramming of adult hepatic cells. Finally, our RNA-seq analysis might be used as an in vivo reference for direct comparison with diseased human tissues such as pancreas dysplasia or agenesis or congenital liver defects.

\section{Materials and methods}

\section{Mouse embryo dissection and FACS sorting}

The transgenic mouse line $\mathrm{Tg}$ (Proxl-EGFP) ${ }^{\text {Gsat/Mmcd }}$ was obtained from the Heintz Laboratory-Gensat Project and generated as previously described (Gong et al. 2003). E8.5 and E10.5 Prox1EGFP-positive embryos were selected and dissected using an epifluorescence stereomicroscope (Discovery V12, Zeiss). Eyebrow knives or tweezers were used for cutting the GFP-positive embryonic regions. The dissected embryonic regions were dissociated by trypsin (trypsin/EDTA $0.25 \%$ solution) digestion into single-cell suspension. To stop the reaction, DMEM (Invitrogen) was added to the cell suspensions and centrifuged at $300 \mathrm{~g}$ at $4^{\circ} \mathrm{C}$, and pellets were suspended and PBS/DEPC-treated. Before sorting, propidium iodide was added to select only live cells, and the cell suspension was filtered through a BD Falcon tube with cell strainer cap (BD 352235). After dead cell exclusion (SSC-A/PI-A), GFP-expressing cells were sorted at $4^{\circ} \mathrm{C}$ using a FACS Aria III flow cytometer (BD Biosciences) using a GFP filter and by setting the gate on the GFP fluorescence intensity. Conditions of sorting were as follows: $70-\mu \mathrm{m}$ nozzle and sheath pressure of 70 psi. Sorted cells were collected directly in TRIzol reagent (Invitrogen) for RNA extraction. Cells isolated by FACS were used for RNA isolation and RNA-seq and subsequently for RT-qPCR validation. Approximately, a total of 10,000 cells per sample were pooled to isolate $100 \mathrm{ng}$ of high-quality total RNA. All animal experimentation was conducted in accordance with the local ethics committee for animal care.

\section{RNA-seq}

RNA-seq was performed using $\sim 70$ ng of total RNA quantified by Agilent RNA 6000 Pico kit (Agilent Technologies). The quality of RNA samples prior to library preparation was determined using an Agilent Bioanalzyer, and only samples with high RIN (RNA integrity number) scores $(>8.5)$ were further processed. Briefly, poly(A) RNA was isolated by two rounds of oligo(dT) 25 Dynabeads (Invitrogen) purification. Purified poly(A) RNA was fragmented for $3.5 \mathrm{~min}$ at $94^{\circ} \mathrm{C}$ using $5 \times$ fragmentation buffer (200 mM Tris-acetate at pH 8.1, 500 mM KOAc, 150 mM MgOA) as described previously (Adamidi et al. 2011). The fragmented RNA was purified by Agencourt RNAClean XP SPRI beads (Agencourt) and converted to first strand cDNA using random hexamer primers (Invitrogen) and SuperScript II reverse transcriptase (Invitrogen) followed by second strand cDNA synthesis with Escherichia coli DNA polymerase I (Invitrogen) and RNase H (Invitrogen) according to the manufacturer's intstructions. The 76-nucleotide (nt) paired-end sequencing library was prepared using New England Biolabs Next DNA Library preparation kit following the Illumina mRNA-Seq library preparation protocol. The minimum necessary number of cycles of amplification was used to minimize amplification biases (15 cycles with no overamplification observed). The quality of cDNA libraries for sequencing was assessed using Agilent Technologies 2100 Bioanalyzer. The prepared sequencing library was subsequently sequenced on Illumina HiSeq for $2 \times 100$ cycles following the standard protocol (Adamidi et al. 2011).

\section{Bioinformatics analysis}

Mapped reads from TopHat (version 1.33) were used as input for Cufflinks (version 1.3.0) (Trapnell et al. 2010) for transcript assembly and differential expression using the University of California at Santa Cruz $\mathrm{mm} 9$ reference annotation. To obtain a working data set for the purposes of comparing transcriptomes 
between different samples, we filtered the Cufflinks gene set for (1) successful deconvolution of fragments per kilobase of exon per million mapped fragments (FPKM), (2) FPKM $>0$ in at least two samples, and (3) lower confidence level of FPKM $>0$ in at least two samples. In the case that multiple Cufflinks genes for one gene symbol existed, Cufflinks genes were further selected for greatest transcript length and highest variability in the FPKM across all samples. This resulted in a nonredundant gene set of 14,053 Cufflinks genes.

Unless otherwise noted, bioinformatic analysis was carried out using R statistical environment (version $\geq 2.15$; http:// www.r-project.org). For the PCA of the nonredundant working data set, a ceiling FPKM value was set as the 90th percentile of all expression values in the data set. The first two principal components exhibited $48 \%$ and $35 \%$ of the total variability for $\mathrm{PC} 1$ and PC2, respectively. The Venn diagram was generated using the R packages limma (Ritchie et al. 2007) and VennDiagram (Adamidi et al. 2011). Enriched GO categories were calculated using the Bioconductor package TopGO (Alexa et al. 2006). In each case, the enrichment of terms in the group FP was compared with all genes in the working data set with annotations in their respective ontologies.

To demonstrate relevance with human pancreatic development, we obtained normalized microarray data from a previously published study of hESC differentiation to pancreatic cells (Micallef et al. 2012). Differential gene expression (at a false discovery rate $[$ FDR] adjusted $P$-value $<0.05$ ) between the differentiated pancreas-like cells ("Nostro protocol") and hESC lines was determined using the R statistical package limma (Smyth 2005). Mouse genes from the RNA-seq experiments were matched to their corresponding human homologs using the NCBI database HomoloGene (http://www.ncbi.nlm.nih.gov/homologene). The RNA-seq data sets have been deposited in NCBI's Gene Expression Omnibus (GEO) and are accessible through accession number GSE40823.

\section{Immunohistochemistry and in situ hybridization}

Mouse embryos were fixed in $4 \%$ paraformaldehyde from $2 \mathrm{~h}$ to overnight at $4^{\circ} \mathrm{C}$. Subsequently, samples were equilibrated in $20 \%$ sucrose solution and embedded in OCT compound (Sakura). In situ hybridization on cryostat sections was done as in Schaeren-Wiemers and Gerfin-Moser (1993). Cryosections (10 $\mu \mathrm{m}$ ) were incubated with TSA (Perkin Elmer) blocking buffer for $1 \mathrm{~h}$ at room temperature and afterward with primary antibodies at an appropriate dilution (Supplemental Table 4). All confocal images were acquired with an LSM 700 confocal laser-scanning microscope (Zeiss).

\section{$R T-q P C R$}

For RNA isolation, embryonic tissues were microdissected, FACS-sorted, and collected in TRIzol reagent (Invitrogen). Subsequently, RNA was extracted from a minimum of 3000 cells according to the manufacturer's instructions (Roche). Briefly, total RNA was resuspended in $10 \mu \mathrm{L}$ of $\mathrm{DEPC} \mathrm{H}_{2} \mathrm{O}$ and processed for reverse transcription using Transcriptor First Strand cDNA Synthesis kit (Roche). A mix of anchored oligo(dT)18 and random hexamer primers was used to generate the cDNA. Thermolabile nuclease from the Real-Time Ready Cell Lysis kit (Roche) was added to the reverse transcription reaction to degrade dsDNA. Real-time PCR reactions were carried out using the SYBR Green Master mix (Roche) on the ABI StepOne Plus system. Succinate dehydrogenase (SDHA) was used as reference gene. All of the values were normalized to the reference gene and calculated using the REST program (Pfaffl et al. 2002). Data were deter- mined in triplets. All experiments were repeated at least three times unless otherwise stated. Primer sequences can be obtained on request.

\section{Xenopus embryo experiments}

Xenopus embryo manipulations and dissections were performed as described (Spagnoli and Brivanlou 2008). The SP64T-xWnt5a plasmid used for microinjection was previously described (Moon et al. 1993). For whole endodermal explant experiments, endodermal cells were microdissected at stage 9 and cultured in $0.1 \times$ MMR in the presence of the indicated recombinant proteins until the desired stage. For anterior endodermal explants, dorsal vegetal cells were microdissected at stage 10 and cultured as above. Ornithine decarboxylase $(O D C)$ was used as a reference gene in Xenopus RT-qPCR analysis. All of the values were normalized to the reference gene and calculated using the REST program (Pfaffl et al. 2002). For luciferase reporter assays in Xenopus, embryos were injected with ATF2-luc (Ohkawara and Niehrs 2011) or TOPFLASH and Renilla-TK plasmid DNA (Clevers and Nusse 2012). Three pools of 15 explants each were lysed with passive lysis buffer and assayed for luciferase activity using the Dual Luciferase system (Promega). Recombinant mouse Wnt5a, Wnt5b, and Wnt3a (R\&D System) proteins were used at the indicated concentrations in both Xenopus and cell culture experiments. All experiments were repeated at least three times unless otherwise stated.

\section{Cell culture experiments}

mESCs (R1 mESC line) were maintained on gelatin-coated plates with mouse embryonic fibroblasts (MEFs) in standard mESC medium: DMEM (Invitrogen), $2 \mathrm{mM}$ glutamax (Invitrogen), $1 \mathrm{mM}$ sodium pyruvate (Invitrogen), $0.1 \mathrm{mM}$ nonessential amino acids (Invitrogen), 15\% fetal bovine serum (FBS) (PAN Biotech), $0.1 \mathrm{mM} \beta$-mercaptoethanol (Sigma), and $1000 \mathrm{U} / \mathrm{mL}$ leukemia inhibitory factor (ESGRO). For differentiation, cultures were MEF-depleted and seeded in mESC medium at high confluency on gelatin-coated dishes. Monolayer differentiation was carried out as described previously (D'Amour et al. 2006; Nostro et al. 2011). Briefly, definitive endoderm medium to day 2 consisted of RPMI medium (Invitrogen) and $0.2 \%$ FBS supplemented with 50 $\mathrm{ng} / \mathrm{mL}$ Activin A and $25 \mathrm{ng} / \mathrm{mL}$ Wnt3a at day 1 and Activin A at day 2. Pancreatic endoderm medium to day 5 consisted of RPMI medium and $2 \%$ FBS supplemented with $3 \mathrm{ng} / \mathrm{mL} \mathrm{Wnt3a}$ and $50 \mathrm{ng} / \mathrm{mL}$ FGF10. Pancreatic progenitor medium to day 8 consisted of DMEM with $1 \%$ (v/v) B27 supplement (Invitrogen), $50 \mu \mathrm{g} / \mathrm{mL}$ ascorbic acid (Sigma), $0.25 \mu \mathrm{M}$ KAAD-cyclopamine (Toronto Research Chemicals), $2 \mu \mathrm{M}$ all-trans retinoic acid (Sigma), and $50 \mathrm{ng} / \mathrm{mL}$ noggin. All recombinant proteins were purchased from $\mathrm{R} \& \mathrm{D}$ System unless otherwise stated. The BAML cells were cultured as previously described (Fougère-Deschatrette et al. 2006).

\section{Western blot analysis}

For Western blot analysis, endodermal cells were lysed as described (Spagnoli and Brivanlou 2008). Immunoblots were incubated with anti-ABC monoclonal antibody, which recognizes the dephosphorylated $\beta$-catenin on Ser37 and Thr41 (Millipore); anti- $\beta$-catenin polyclonal antibody (Santa Cruz Biotechnology); anti-pJNK monoclonal antibody (Santa Cruz Biotechnology); and anti- $\alpha$-tubulin monoclonal antibody (Sigma) and analyzed using the LI-COR Odyssey system.

\section{Statistical tests}

All results are expressed as mean \pm standard errors. The significance of differences between groups was evaluated with 
Student's $t$-test. $P<0.05$ was considered statistically significant. Statistical tests relevant to the RNA-seq data set analysis are described in the "Bioinformatic Analysis" section.

\section{Acknowledgments}

We thank W. Birchmeier for discussion and critical reading of the manuscript, and all members of the Spagnoli, Andrade, and Chen laboratories for helpful discussions. We thank the Max Delbrück Center FACS Core Facility and Dr. Hans-Peter Rahn for support and technical assistance. We are grateful to T. Uemura for the Celsr2 antibody, Y. Minami for the Ror2 antibody, and C. Niehrs for the ATF2-luc plasmid. This research was supported by institutional funds from the Helmholtz Association and the Bundesministerium für Bildung und Forschung (BMBF). F.M.S. is supported by the European Research Council (ERC)-Starting Hepatopancreatic Grant 243045.

\section{References}

Adamidi C, Wang Y, Gruen D, Mastrobuoni G, You X, Tolle D, Dodt M, Mackowiak S, Gogol-Doering A, Oenal P, et al. 2011. De novo assembly and validation of planaria transcriptome by massive parallel sequencing and shotgun proteomics. Genome Res 21: 1193-1200.

Alexa A, Rahnenführer J, Lengauer T. 2006. Improved scoring of functional groups from gene expression data by decorrelating GO graph structure. Bioinformatics 22: 1600-1607.

Angers S, Moon R. 2009. Proximal events in Wnt signal transduction. Nat Rev Mol Cell Biol 10: 468-477.

Burke Z, Oliver G. 2002. Prox1 is an early specific marker for the developing liver and pancreas in the mammalian foregut endoderm. Mech Dev 118: 147-155.

Chalmers A, Slack J, Beck C. 2000. Regional gene expression in the epithelia of the Xenopus tadpole gut. Mech Dev 96: 125128.

Chen A, Borowiak M, Sherwood R, Kweudjeu A, Melton D. 2013. Functional evaluation of ES cell-derived endodermal populations reveals differences between Nodal and Activin A-guided differentiation. Development 140: 675-686.

Chung W, Shin C, Stainier D. 2008. Bmp2 signaling regulates the hepatic versus pancreatic fate decision. Dev Cell 15: 738748.

Clevers H, Nusse R. 2012. Wnt/ $/$-catenin signaling and disease. Cell Mol Life Sci 149: 1192-1205.

D'Amour K, Bang A, Susan Eliazer S, Kelly O, Agulnick A, Smart N, Moorman M, Kroon E, Carpenter M, Baetge E. 2006. Production of pancreatic hormone-expressing endocrine cells from human embryonic stem cells. Nat Biotechnol 24: 1392-1401.

Dequéant M, Glynn E, Gaudenz K, Wahl M, Chen J, Mushegian A, Pourquié O. 2006. A complex oscillating network of signaling genes underlies the mouse segmentation clock. Science 314: 1595-1598.

Deutsch G, Jung J, Zheng M, Lóra J, Zaret KS. 2001. A bipotential precursor population for pancreas and liver within the embryonic endoderm. Development 128: 871-881.

Dichmann D, Harland R. 2011. Nkx6 genes pattern the frog neural plate and Nkx6.1 is necessary for motoneuron axon projection. Dev Biol 349: 378-386.

Dinger M, Amaral P, Mercer T, Pang K, Bruce S, Gardiner B, Askarian-Amiri M, Ru K, Soldà G, Simons C, et al. 2008. Long noncoding RNAs in mouse embryonic stem cell pluripotency and differentiation. Genome Res 18: 1433-1445.

Fougère-Deschatrette $C$, Imaizumi-Scherrer $T$, Strick-Marchand H, Morosan S, Charneau P, Kremsdorf D, Faust D, Weiss M.
2006. Plasticity of hepatic cell differentiation: Bipotential adult mouse liver clonal cell lines competent to differentiate in vitro and in vivo. Stem Cells 24: 2098-2109.

Gong S, Zheng C, Doughty M, Losos K, Didkovsky N, Schambra U, Nowak N, Joyner A, Leblanc G, Hatten M, et al. 2003. A gene expression atlas of the central nervous system based on bacterial artificial chromosomes. Nature 425: 917-925.

Grapin-Botton A, Melton D. 2000. Endoderm development: From patterning to organogenesis. Trends Genet 16: 124-130.

Grigoryan T, Wend P, Klaus A, Birchmeier W. 2008. Deciphering the function of canonical Wnt signals in development and disease: Conditional loss- and gain-of-function mutations of $\beta$-catenin in mice. Genes Dev 22: 2308-2341.

Grumolato L, Liu G, Mong P, Mudbhary R, Biswas R, Arroyave R, Vijayakumar S, Economides A, Aaronson S. 2010. Canonical and noncanonical Wnts use a common mechanism to activate completely unrelated coreceptors. Genes Dev 24: 2517-2530.

Ho H, Susman M, Bikoff J, Ryu Y, Jonas A, Hu L, Kuruvilla R, Greenberg M. 2012. Wnt5a-Ror-Dishevelled signaling constitutes a core developmental pathway that controls tissue morphogenesis. Proc Natl Acad Sci 109: 4044-4051.

Hou J, Charters A, Lee S, Zhao Y, Wu M, Jones S, Marra M, Hoodless P. 2007. A systematic screen for genes expressed in definitive endoderm by serial analysis of gene expression (SAGE). BMC Dev Biol 7: 92.

Iimura T, Denans N, Pourquié O. 2009. Establishment of Hox vertebral identities in the embryonic spine precursors. Curr Top Dev Biol 88: 201-234.

Karner C, Chirumamilla R, Aoki S, Igarashi P, Wallingford J, Carroll T. 2009. Wnt9b signaling regulates planar cell polarity and kidney tubule morphogenesis. Nat Genet 41: 793799.

Kikuchi A, Yamamoto H, Sato A. 2009. Selective activation mechanisms of Wnt signaling pathways. Trends Cell Biol 19: 119-129.

Kim H, Schleiffarth J, Jessurun J, Sumanas S, Petryk A, Lin S, Ekker S. 2005. Wnt5 signaling in vertebrate pancreas development. BMC Biol 24: 23.

Lee C, Friedman J, Fulmer J, Kaestner K. 2005. The initiation of liver development is dependent on Foxa transcription factors. Nature 435: 944-947.

Li Y, Rankin S, Sinner D, Kenny A, Krieg P, Zorn A. 2008. Sfrp5 coordinates foregut specification and morphogenesis by antagonizing both canonical and noncanonical Wnt11 signaling. Genes Dev 22: 3050-3063.

Micallef S, Li X, Schiesser JV, Hirst CE, Yu QC, Lim SM, Nostro MC, Elliott DA, Sarangi F, Harrison LC, et al. 2012. INSGFP/w human embryonic stem cells facilitate isolation of in vitro derived insulin-producing cells. Diabetologia 55: 694-706.

Miki R, Yoshida T, Murata K, Oki S, Kume K, Kume S. 2012. Fate maps of ventral and dorsal pancreatic progenitor cells in early somite stage mouse embryos. Mech Dev 128: 597609.

Mitiku N, Baker J. 2007. Genomic analysis of gastrulation and organogenesis in the mouse. Dev Cell 13: 897-907.

Moon R, Campbell R, Christian J, McGrew L, Shih J, Fraser S. 1993. Xwnt-5A: A maternal Wnt that affects morphogenetic movements after overexpression in embryos of Xenopus laevis. Development 119: 97-111.

Nostro M, Sarangi F, Ogawa S, Holtzinger A, Corneo B, Li X, Micallef S, Park I, Basford C, Wheeler M, et al. 2011. Stagespecific signaling through TGF $\beta$ family members and WNT regulates patterning and pancreatic specification of human pluripotent stem cells. Development 138: 861-871. 
Ober E, Verkade H, Field H, Stainier D. 2006. Mesodermal Wnt $2 \mathrm{~b}$ signalling positively regulates liver specification. Nature 442: 688-691.

Ohkawara B, Niehrs C. 2011. An ATF2-based luciferase reporter to monitor non-canonical Wnt signaling in Xenopus embryos. Dev Dyn 240: 188-194.

Pfaffl MW, Horgan GW, Dempfle L. 2002. Relative expression software tool (REST) for group-wise comparison and statistical analysis of relative expression results in real-time PCR. Nucleic Acids Res 30: e36.

Puri S, Hebrok M. 2010. Cellular plasticity within the pancreas-lessons learned from development. Dev Cell 18: 342356.

Ritchie M, Silver J, Oshlack A, Holmes M, Diyagama D, Holloway A, Smyth G. 2007. A comparison of background correction methods for two-colour microarrays. Bioinformatics 23: 27002707.

Rossant J. 2011. The impact of developmental biology on pluripotent stem cell research: Successes and challenges. Dev Cell 21: 20-23.

Schaeren-Wiemers N, Gerfin-Moser A. 1993. A single protocol to detect transcripts of various types and expression levels in neural tissue and cultured cells: In situ hybridization using digoxigenin-labelled cRNA probes. Histochemistry 100: $431-440$.

Slack JM. 2007. Metaplasia and transdifferentiation: From pure biology to the clinic. Nat Rev Mol Cell Biol 8: 369-378.

Smyth GK. 2005. Limma: Linear models for microarray data. In Bioinformatics and computational biology solutions using $R$ and bioconductor (ed. R Gentleman et al.), pp. 397-420. Springer, New York.

Spagnoli FM. 2007. From endoderm to pancreas: A multistep journey. Cell Mol Life Sci 64: 2378-2390.

Spagnoli FM, Brivanlou AH. 2008. The Gata5 target, TGIF2, defines the pancreatic region by modulating BMP signals within the endoderm. Development 135: 451-461.

Trapnell C, Williams B, Pertea G, Mortazavi A, Kwan G, van Baren M, Salzberg S, Wold B, Pachter L. 2010. Transcript assembly and quantification by RNA-seq reveals unannotated transcripts and isoform switching during cell differentiation. Nat Biotechnol 28: 511-515.

Tremblay KD, Zaret KS. 2005. Distinct populations of endoderm cells converge to generate the embryonic liver bud and ventral foregut tissues. Dev Biol 280: 87-99.

Tumbar T, Guasch G, Greco V, Blanpain C, Lowry W, Rendl M, Fuchs E. 2004. Defining the epithelial stem cell niche in skin. Science 303: 359-363.

van Noort M, Meeldijk J, van der Zee R, Destree O, Clevers H. 2002. Wnt signaling controls the phosphorylation status of ß-catenin. J Biol Chem 277: 17901-17905.

Vichas A, Zallen J. 2011. Translating cell polarity into tissue elongation. Semin Cell Dev Biol 22: 858-864.

Wallingford J. 2012. Planar cell polarity and the developmental control of cell behavior in vertebrate embryos. Annu Rev Cell Dev Biol 28: 627-653.

Wandzioch E, Zaret K. 2009. Dynamic signaling network for the specification of embryonic pancreas and liver progenitors. Science 324: 1707-1710.

Wang Q, Piotrowska K, Ciemerych M, Milenkovic L, Scott M, Davis R, Zernicka-Goetz M. 2004. A genome-wide study of gene activity reveals developmental signaling pathways in the preimplantation mouse embryo. Dev Cell 6: 133-144.

Wang P, Rodriguez R, Wang J, Ghodasara A, Kim S. 2011. Targeting SOX17 in human embryonic stem cells creates unique strategies for isolating and analyzing developing endoderm. Cell Stem Cell 8: 335-346.
Watanabe T, Nakagawa K, Ohata S, Kitagawa D, Nishitai G, Seo J, Tanemura T, Shimizu N, Kishimoto H, Wada T, et al. 2002. SEK1/MKK4-mediated SAPK/JNK signaling participates in embryonic hepatoblast proliferation via a pathway different from NF-кB-induced anti-apoptosis. Dev Biol 250: 332-347.

Zaret K. 2008. Genetic programming of liver and pancreas progenitors: Lessons for stem-cell differentiation. Nat ReV Genet 9: 329-340.

Zhang Z, Rankin S, Zorn A. 2013. Different thresholds of WntFrizzled 7 signaling coordinate proliferation, morphogenesis and fate of endoderm progenitor cells. Dev Biol 378: 1-12. 


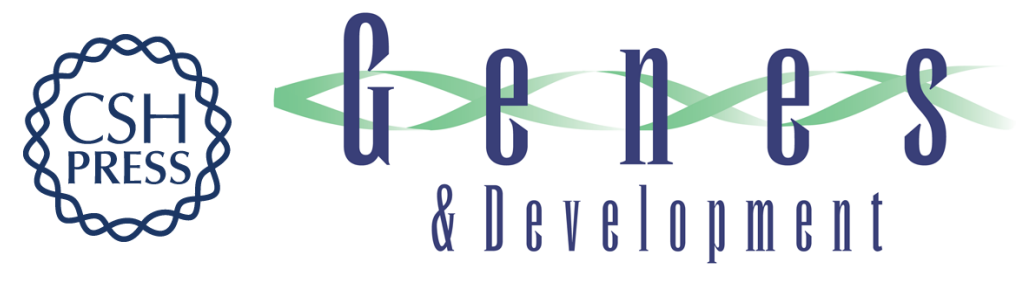

\section{Mutually exclusive signaling signatures define the hepatic and pancreatic progenitor cell lineage divergence}

Elisa Rodríguez-Seguel, Nancy Mah, Heike Naumann, et al.

Genes Dev. 2013, 27:

Access the most recent version at doi:10.1101/gad.220244.113

\section{Supplemental http://genesdev.cshlp.org/content/suppl/2013/09/06/27.17.1932.DC1 Material}

References

This article cites 53 articles, 15 of which can be accessed free at: http://genesdev.cshlp.org/content/27/17/1932.full.html\#ref-list-1

Creative This article is distributed exclusively by Cold Spring Harbor Laboratory Press for the first Commons six months after the full-issue publication date (see

License http://genesdev.cshlp.org/site/misc/terms.xhtml). After six months, it is available under a Creative Commons License (Attribution-NonCommercial 3.0 Unported), as described at http://creativecommons.org/licenses/by-nc/3.0/.

Email Alerting Receive free email alerts when new articles cite this article - sign up in the box at the top Service right corner of the article or click here.

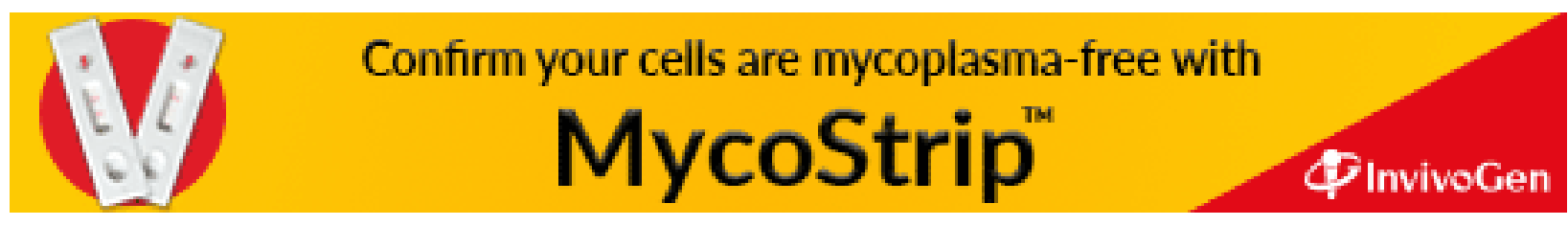

